\title{
NEAMS M2 Milestone Report: Development of a UO2 Grain Size Model using Multicale Modeling and Simulation
}

Michael Tonks, Yongfeng Zhang, and Xianming Bai

June 2014

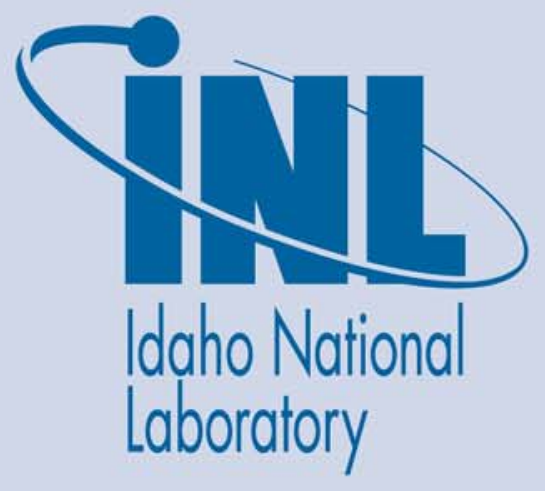

The INL is a U.S. Department of Energy National Laboratory operated by Battelle Energy Alliance 
INL/EXT-14-32450

\title{
NEAMS M2 Milestone Report: Development of a UO2 Grain Size Model using Multicale Modeling and Simulation
}

Michael Tonks, Yongfeng Zhang, and Xianming Bai

June 2014

\author{
Idaho National Laboratory \\ Idaho Falls, Idaho 83415
}

http://www.inl.gov

Prepared for the

U.S. Department of Energy

Office of Nuclear Energy

Under DOE Idaho Operations Office

Contract DE-AC07-05ID14517 


\section{NEAMS M2 Milestone Report: \\ Development of a $\mathrm{UO}_{2}$ Grain Size Model using Multicale Modeling and Simulation}

Michael Tonks, Yongfeng Zhang, and Xianming Bai

June 26, 2014 


\section{Contents}

1 Introduction $\quad 1$

1.1 Fuel Performance Modeling Based on Microstructure Rather Than Burnup 1

$1.2 \mathrm{UO}_{2}$ Grain Size . . . . . . . . . . . . . . . . . . 3

2 GB Mobility 5

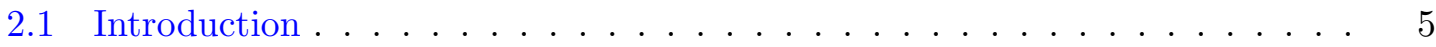

2.2 Models . . . . . . . . . . . . . . . . . . . . 6

2.2.1 Curvature-driving GB motion . . . . . . . . . . . 6

2.2.2 GB motion under zero driving force . . . . . . . . . . . . 7

2.3 Method ............................ 7

2.4 Results . . . . . . . . . . . . . . . . . . . . . . 9

2.5 Discussion . . . . . . . . . . . . . . . . . . . . . . 10

3 Driving Force 14

3.1 Introduction . . . . . . . . . . . . . . . . . . . . . 14

3.2 Grain Boundary Energy Calculation . . . . . . . . . . . . . . . 15

3.3 Verification of the Temperature Gradient Driving Force Equation . . . . . . 17

3.4 Investigation of Significance of Temperature Gradient Driving Force . . . . 26

4 Resistive Force $\quad 32$

4.1 Sintered Porosity Model . . . . . . . . . . . . . . . . . . . . . . 34

4.2 GB Bubble Pinning Model . . . . . . . . . . . . . . . . . . . 34

4.2.1 Pore/GB Interaction Phase Field Model . . . . . . . . . . . . . . . . 36

4.2.2 Investigation of the Analytical Model . . . . . . . . . . . . . . 39

5 Bison Implementation $\quad 42$

6 Conclusion $\quad 44$ 


\section{Chapter 1}

\section{Introduction}

\subsection{Fuel Performance Modeling Based on Microstructure Rather Than Burnup}

One of the primary goals of the Fuels Product Line (FPL) in the Nuclear Energy Advanced Modeling and Simulation (NEAMS) program is to develop mechanistic materials models that model the material behavior based on microstructure rather than burnup. These models will be the basis of a more predictive fuel performance capability that is being obtained through the development of the MOOSE-BISON-MARMOT (MBM) suite of codes.

In this approach, as summarized in Tonks et al. [29], the current state of the microstructure is represented by a series of microstructure variables within the BISON fuel performance code, as illustrated in Fig. 1.1. These include attributes such as the average grain size, or the intragranular porosity. Models must be developed to describe the evolution of these variables as a function of temperature, stress, and neutron flux, as well as other microstructure variables. In addition, models are required to define how the various material properties vary with respect to the microstructure variables. This approach bases the model on the current state of the microstructure rather than the burnup, which is not a unique measure of the history of the material. In addition, these relationships defining the microstructure are developed as physics-based models rather than empirical fits, allowing the models to be extrapolated outside of the bounds of data used for validation.

While this approach provides the predictive capability required for the next generation of fuel performance codes, it can be a difficult process requiring significant information about the material behavior within the reactor. While some of this information can be obtained experimentally, this hard to obtain experimental data is supplemented using multiscale 


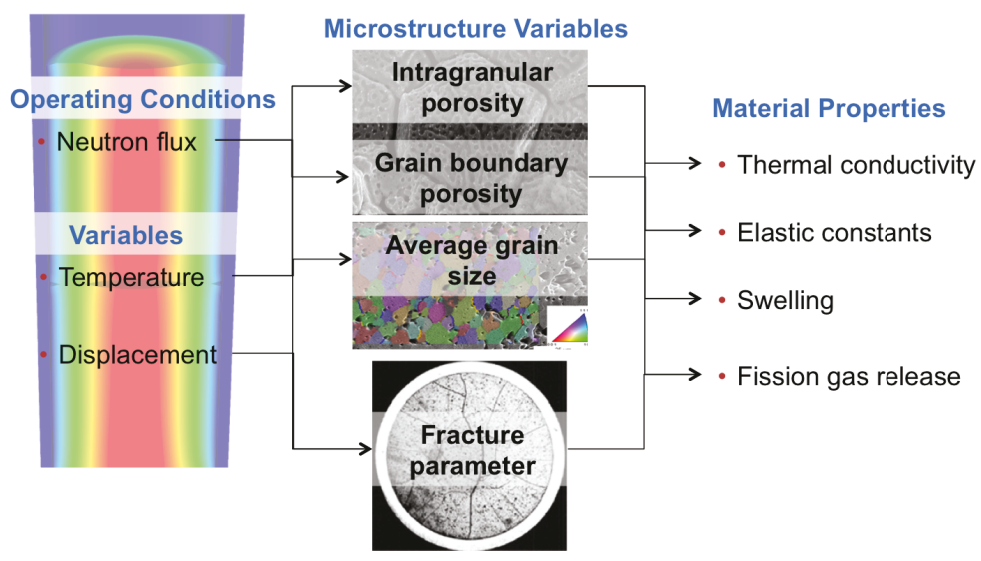

Figure 1.1: Illustration of the NEAMS FPL approach to develop predictive materials models for fuel performance based on microstructure rather than burnup.

modeling and simulation, as shown in Fig. 1.2. First principle density functional theory calculations (representing 100's of atoms) are used to identify critical bulk mechanisms and to determine bulk properties. Atomistic molecular dynamics (MD) simulations are used to identify interfacial mechanisms, as well as dynamic and interfacial properties. Mesoscale simulations using INL's MARMOT code [28] predict the microstructure evolution as well as the effective properties (thermal conductivity, elastic modulus, etc) of the microstructure. This mesoscale information provides guidance throughout the development of the physicsbased models required to predict the evolution of the microstructure variables and how they impact the material properties within BISON.

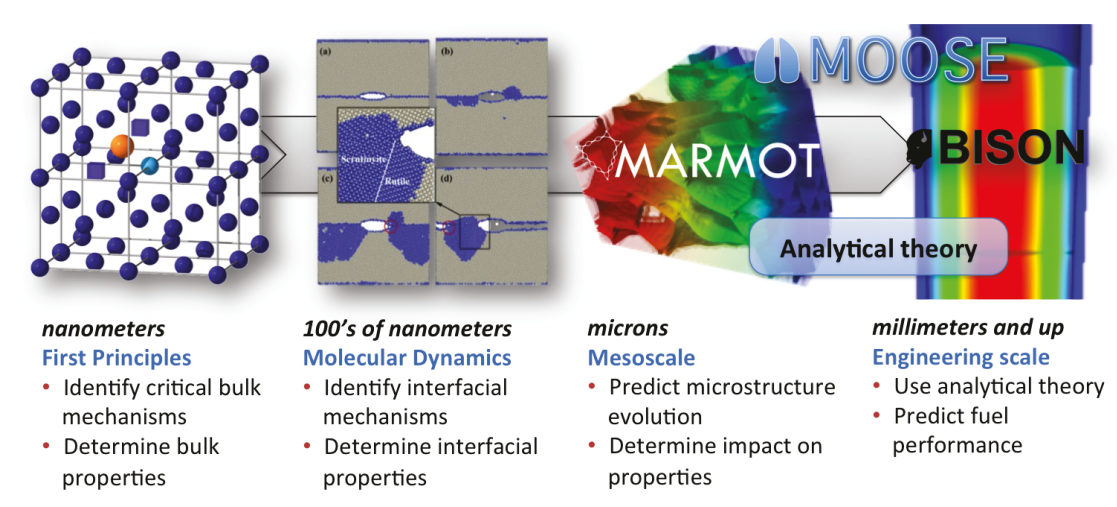

Figure 1.2: Illustration of the NEAMS FPL multiscale approach to develop microstructurebased materials models. 
In this report, we illustrate this development process by summarizing the various steps that have been carried out by researchers at Idaho National Laboratory (INL) to develop a model for the average grain size in $\mathrm{UO}_{2}$.

\section{$1.2 \quad \mathrm{UO}_{2}$ Grain Size}

The grain size of $\mathrm{UO}_{2}$ fuel pellets typically begin with an average grain size of $10 \mu \mathrm{m}$. However, during reactor operation, the temperature near the center of the pellet rises above $1500 \mathrm{~K}$. At these temperatures the mobility of the grain boundaries (GBs) rise sufficiently for them to migrate to reduce the overall free energy of the system. Smaller grains tend to disappear and larger grains tend to grow, such that the average grain size increases with time. At higher burnups, the material near the colder out rim of the fuel receives sufficient damage to cause grain subdivision, resulting in very small grains and large bubbles. This structure is typically called high burnup structure (HBS). Both the grain growth in the hot portion of the fuel and HBS formation in the rim region significantly impact the fuel performance. However, in this work we only consider the impact of grain growth.

The increasing grain size within the fuel is important because it significantly impacts the swelling, fission gas release, and creep. In fact, a recent study found that the swelling model in BISON is quite sensitive to the grain radius [21]. For this reason, it is critical to accurately predict the average grain radius. However, due to the large temperature gradient and differences in the local microstructure, the grain radius will vary throughout the pellet. While models do exist to predict the change in the grain radius within the fuel, they are primarily empirical [2] or rely on a large number of parameters that are determined by fitting to data [34]. In this work, we develop a mechanistic model for grain growth in which the various parameters and terms are developed using multiscale modeling and simulation.

The velocity of a migrating GB can be approximated by the simple model [8]

$$
v_{G B}=M_{G B}\left(P_{D F} \pm P_{r}\right),
$$

where $M_{G B}$ is the GB mobility and $P_{D F}$ is the driving force and $P_{r}$ is a resistive force that opposes the driving force and is due to secondary particles or pores. Both $P_{D F}$ and $P_{r}$ take the form of energy per unit volume. The driving force is due to the drive of the system to reduce the overall energy, and various driving forces can be active in a given material. The velocity equation can be used to give the general form for an equation defining the change in the average grain size $\dot{D}$, according to

$$
\dot{D}=2 M_{G B}\left(P_{D F}-P_{r}\right),
$$

where we assume that the change in the grain radius $\dot{R}=v_{G B}$ and that $D=2 R$. This equation provides the basis for a physics-based model for the average grain radius in the 
fuel. However, to complete this model, we require more information about each of its individual terams, i.e. the GB mobility $M_{G B}$, the driving force $P_{D F}$, and the resistive force $P_{r}$. To determine this information, we use simulation methods at the atomistic and mesoscale. For the remainder of this document, we discuss our approach for each term in the following sections. 


\section{Chapter 2}

\section{Grain Boundary Mobility}

\section{$2.1 \quad$ Introduction}

Grain boundaries migrate under various driving forces which governs the thermodynamical aspect of grain growth. The kinetic aspect, i.e., how fast of a specific GB can migrate under a certain driving force, is controlled by the mobility $M$. For $\mathrm{UO}_{2}$, the mobility has been measured experimentally using sintered samples by analyzing the grain growth data [2]. In the experiments the samples contains impurities and sintering porosities at different levels. The presence of impurity strongly effects the GB mobility. Even at the concentration of hundred of atomic part-per-million (APPM), the impurities may reduce the mobility by several orders $[19,14]$. On the other hand, the porosities exert resistive force on GBs which counter balances the curvature driving forces (see Chapter 4 for more details). As a result, the measured mobilities in general differ from the intrinsic mobility of $\mathrm{UO}_{2}$, and they apply to certain specific samples only. Therefore, it would be useful to get the intrinsic mobility for pure $\mathrm{UO}_{2}$ which will be a reference for understanding the experimental results. A model that describes the impurity drag effect will then be needed to give the mobility with various impurity levels.

In this Chapter, molecular dynamics (MD) simulations will be used to obtained mobilities for representative GBs in $\mathrm{UO}_{2}$. For the relevance we will focus on curvature driving force which has been determined in Chapter 3 as the primary reason for grain growth. In addition, we introduce the random-walk method as well which gives the mobility under no driving force to check if the mobility is dependent on the type of driving forces. The results obtained by MD are further compared the experimental measurements to elucidate the impurity drag. At the end, a theoretical model will be implemented to bridge the gap between MD and experiments. 


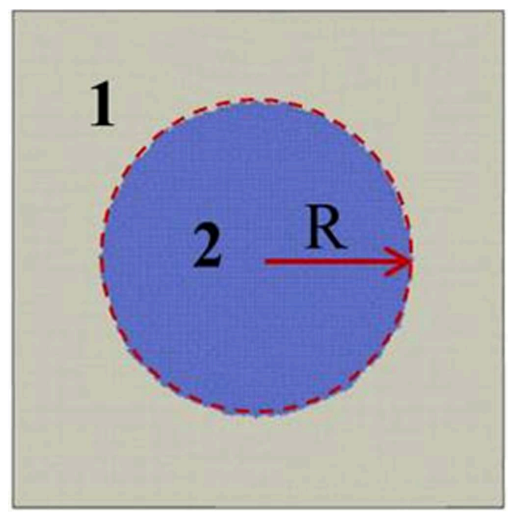

(a)

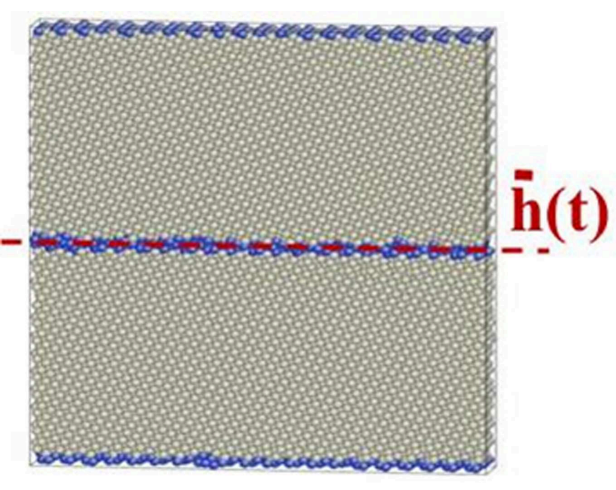

(b)

Figure 2.1: (a) The circular-embedded model for GB migration under curvature driving force, with atoms in grain 1 colored in gray, and those in grain 2 in blue; (b) the bicrystal model for GB migration under zero driving force, with blue atoms representing those in GBs, and others in gray. The dash lines depict the GBs in the models.

\subsection{Models}

\subsubsection{Curvature-driving GB motion}

For the curvature-driving GB migration, we use a circular-embedded model as shown in Fig. 2.1(a). This methods has been shown able to do self-check on any grain size effect, and thus is a better choice than the thumb shape geometry [30]. In this model, a circular grain 2 is embedded into a matrix (grain 1). To reduce the total GB area and thus energy, the grain 2 will shrink at a velocity given by:

$$
v=P M=\frac{d R}{d t}=\frac{M \sigma_{G B}}{R}
$$

Thus we have:

$$
\frac{d R^{2}}{2 d t}=M \sigma_{G B}
$$

Using $A=\pi R^{2}$, with $A$ being the projected area of grain 2, Eq. 2.2 becomes:

$$
\frac{d A}{d t}=M \sigma_{G B}=2 \pi M^{*}
$$

Therefore, the area of grain 2 will shrink proportionally to the product of mobility and GB energy, or the reduced mobility $M^{*}$. Knowledge on GB energy, usually assumed to be a constant, is needed to further derive the mobility $M$. Note that in this method, the average reduced mobility for a family of tilt GBs with the same misorientation angle but different inclination angles, rather than that for a specific GB, is calculated . 


\subsubsection{GB motion under zero driving force}

The bicrystal model as shown in Fig. 2.1(b) is used to calculate the GB mobility under zero driving force. This method calculates the mobility, not the reduced mobility, for a specific GB. As the GBs are flat with zero curvature, no driving force exists for Gb migration globally. However, due to thermal fluctuation, local curvature may still develop stochastically at finite temperature so that GB migrates locally. Consequently, the average GB position $\bar{h}(t)$ will fluctuate in a way similar to one-dimensional Brownian motion [33]. The mean-square-displacement of the average GB position increases linearly with time by:

$$
<\bar{h}^{2}>=2 D t
$$

and the mobility is given by

$$
M=\frac{2 D A_{G B}}{K_{B} T}
$$

In Eq. 2.5, $D$ is the effective diffusivity; $A_{G B}$ is the GB area; $K_{B}$ is Boltzmann constant; $T$ is temperature.

\subsection{Method}

We use the LAMMPS simulation package [23] and the Basak potential [5] for the simulations. For the curvature driving force, a simulation cell as shown in Fig. 2.1(a) is constructed, with the tilt angle between grain 1 and 2 being $45^{\circ}$. The tilt axis is $\langle 100\rangle$. The simulation cell, quasi-2D in geometry, is periodic in all three axial directions. The dimensions of the matrix are $282.4 \AA$ along the two in-plane directions ( $X$ and $Y$ ), and about $22.6 \AA$ along the $Z(\langle 100\rangle)$ direction. The number of total atoms in the simulation system is 119808 . After relaxing the system at room temperature, the system temperature is then brought to constant temperatures from $2100 \mathrm{~K}$ to $3000 \mathrm{~K}$, one simulation every $50 \mathrm{~K}$. The system pressure is maintained at zero to accommodate thermal expansion. To monitor the projected area, we use the orientation parameter [38] to track the number of atoms $N_{2}$ in grain 2. The area A is then estimated by $A=\Omega N_{2} / L_{z}$. Here $\Omega$ and $l_{z}$ are the atomic volume and the simulation cell dimension along the $Z$ axis at a given temperature respectively.

For the random walk method, five different coincidence-site-lattice (CSL, or $\Sigma$ ) GBs have been used for mobility calculations, using the bicrystal model shown in Fig 2.1(b). These GBs are the $\langle 110\rangle \Sigma 3\{111\}$ coherent twin, $\langle 100\rangle \Sigma 5\{310\}$ symmetric tilt, $\langle 100\rangle \Sigma 5\{310\}$ symmetrical twist, $\langle 100\rangle \Sigma 29\{520\}$ symmetric tilt, and $\langle 100\rangle \Sigma 25\{710\}$ symmetric tilt boundaries. These GBs have been used for the simulations verifying the thermal-gradient driving

force in Chapter 3. The simulation cells are quasi-2D and periodic along all three axes. The 
number of atoms varies slightly with the type of GBs. For the two in-plane dimensions, the length is about $178.4 \AA$ along the GB line, and about $215.2 \AA$ along the GB normal. The third dimension is about $22.6 \stackrel{A}{A}$. Again, the bicrystal simulation cells are annealed at temperatures from $2500 \mathrm{~K}$ to $3000 \mathrm{~K}$, one data point every $100 \mathrm{~K}$, using the NPT ensemble from LAMMPS. Due to the absence of a driving force, no meaningful results can be obtained at temperatures below $2500 \mathrm{~K}$ using this method. To track the GB position, the potential energy profile for U-ions is plotted along the GB normal direction, and the peaks are located at the GB positions.

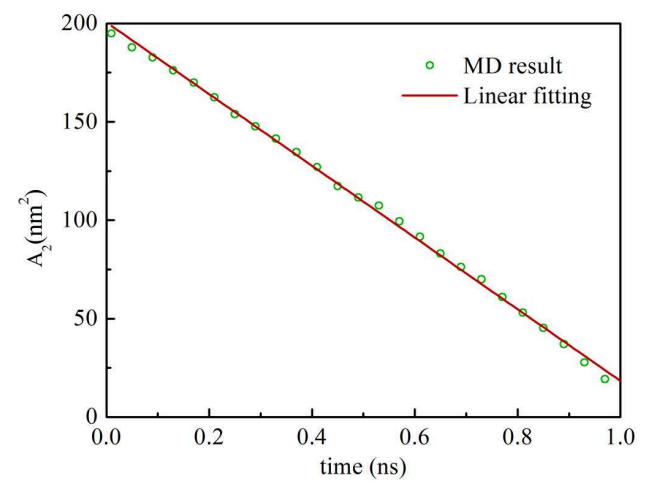

(a)

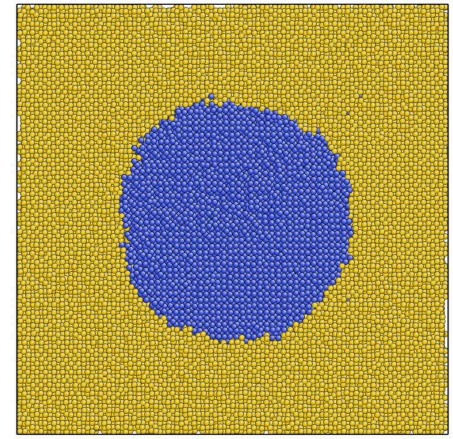

(b)

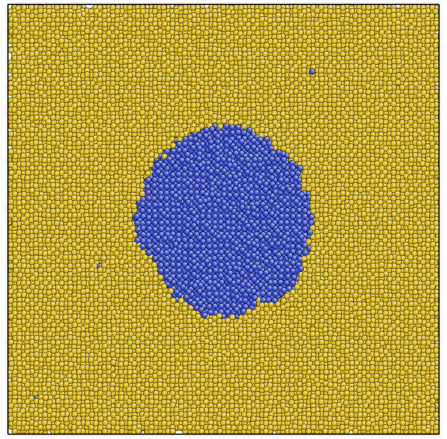

(c)

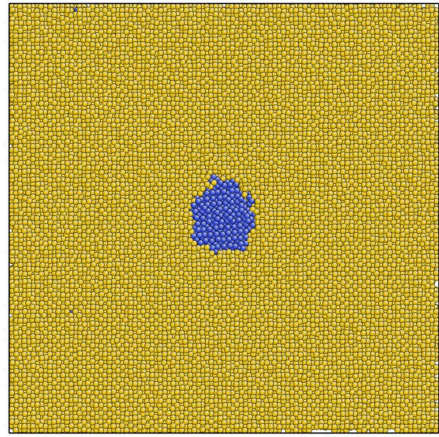

(d)

Figure 2.2: (a) Projected area of grain 2 as a function of time as $2700 \mathrm{~K}$; and the corresponding atomic configurations of the simulation cell at (b) 0.0 , (c) 0.5 and (d) $1.0 \mathrm{~ns}$. In (b)-(d) the atoms are colored by the orientation parameter so that those in grain 1 are in yellow and grain 2 in blue. 


\subsection{Results}

For the curvature-driving GB migration, the reduced mobility can be obtained by plotting the area of grain 2 against time. As an example, the GB migration at $2700 \mathrm{~K}$ is demonstrated in Fig. 2.2. Driving by the GB energy, the grain 2 keeps shrinking with time, as shown in Figs. 2.2(b)-2.2(d). Initially, the grain radius is about $10 \mathrm{~nm}$, and it becomes about $1.0 \mathrm{~nm}$ within $1.0 \mathrm{~ns}$. During migration, the grain 2 roughly maintains a circular shape. The project area of grain 2 reduces linearly with time, as shown in Fig. 2.2(a). The slope obtained by linear fitting gives the reduced mobility at this temperature.

Assuming harmonic behavior and constant GB energy, the reduced mobility is expected to follow the Arrhenius relationship with temperature, i.e., $M^{*}=M_{0}^{*} \exp \left(-E^{m} / K_{B} T\right)$. Here $E^{m}$ is the migration barrier and $M_{0}^{*}$ is the prefactor, both constant over temperature. As shown in Fig. 2.4, the logarithm reduced mobility displays two distinct slopes at two different temperature ranges. Below $2300 \mathrm{~K}$, linear fitting gives a migration barriers of 3.06 $\mathrm{eV}$, and it is given as $1.45 \mathrm{eV}$ beyond $2300 \mathrm{~K}$. This dramatic change in migration barrier suggest that thre might be some change in the GB structure or the migration mechanisms. It has been well known that fluorite phase ceramics experience a Bredig transition at a temperature of about $0.8 \mathrm{~T}_{m}$, the melting point. At temperature beyond the transition point, the self-diffusion of anions is substantially enhanced, indicating an instability of the anion sub-lattice. Using the Basak potential, it was shown that this Bredig transition starts at about $2300 \mathrm{~K}$ in $\mathrm{UO}_{2}[5]$. Therefore, one possible explanation of the result in Fig. 2.4 is that the GB migration barrier is reduced by the occurrence of Bredig transition. Further simulations are being performed to verify this point.

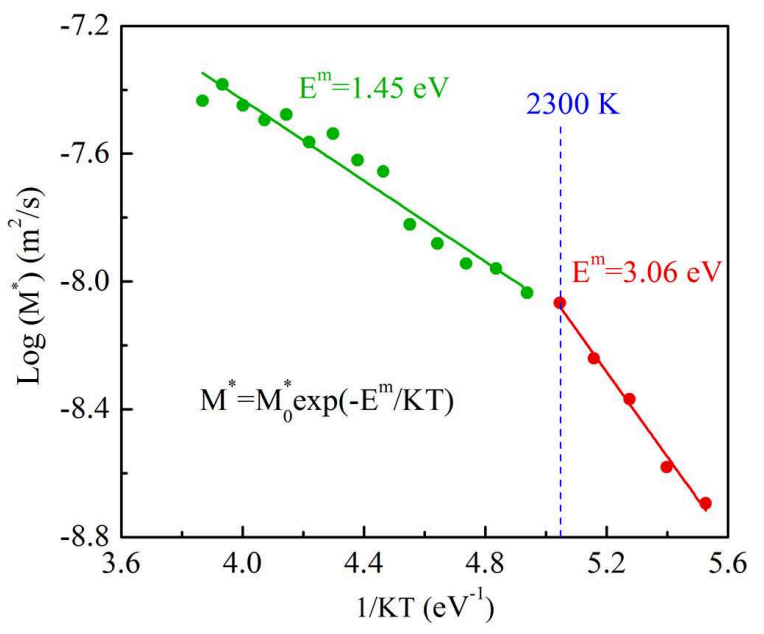

Figure 2.3: Logarithm reduced mobility of the $\langle 100\rangle 45^{\circ}$ tilt GBs as a function of inverse temperature. The lines show linear fittings at different temperature ranges. 
The results obtained with the random-walk method are shown in Fig. 2.4. Owing to the absence of a driving force, high temperature and long simulation time are required to get reliable statistics on the mean-square-displacement (MSD). Meaningful results are obtained at temperatures above $2500 \mathrm{~K}$ only. As a given temperature, the MSD of the average GB position increases linearly with time, and the slopes gives the mobility. As an example, the MSD profile for the $\langle 100\rangle \Sigma 5\{310\}$ GB at $2700 \mathrm{~K}$ is shown in Fig. 2.4(a).

As shown in Fig. 2.4(b), the mobilities of $\mathrm{UO}_{2}$ GBs are highly anisotropic regarding GB characters. Among the five GBs simulated, no sufficient GB fluctuation can be detected for the $\langle 100\rangle \Sigma 25\{710\}$ symmetric tilt GB and the $\langle 110\rangle \Sigma 3\{111\}$ coherent twin to establish the MSD profile. For the other three GBs, the $\langle 100\rangle \Sigma 5\{310\}$ symmetric tilt and the $\langle 100\rangle \Sigma 29\{520\}$ symmetric tilt GBs exhibit about the same mobilities, which are also similar the that for the $\langle 100\rangle 45^{\circ}$ tilt GBs at high temperatures. The mobility for the $\langle 100\rangle \Sigma 5\{310\}$ symmetric twist GB is the lowest among the three.

On the other hand, the GB mobility does not show a strong, or if any, dependence on the driving force. Taking the $\langle 100\rangle \Sigma 5\{310\}$ symmetric tilt GB as example, the results obtained with the random-walk and the thermal-gradient methods are very close to each other (Fig. 2.4(b)). Note that in the thermal-gradient method, some uncertainties exist in determining the GB width, the entropy difference between bulk and GBs, and the atomic volume at GBs. Moreover, the curvature-driving method also gives similar mobility for the $\langle 100\rangle 45^{\circ}$ tilt GBs. Note that both the $\langle 100\rangle \Sigma 5\{310\}$ and the $\langle 100\rangle 45^{\circ}$ tilt GBs are of high energy and high misorientation angle. Therefore, it seems that there is an intrinsic mobility for high-angle-high-energy $\mathrm{GBs}$ in $\mathrm{UO}_{2}$ which is independent on driving forces.

Due to the high temperatures required for the random-walk method, no transition in GB migration barriers over temperature can be established using this method. However, we did notice that at high temperature like 2900 and $3000 \mathrm{~K}$, GBs experience substantial widening compared to that at $2500 \mathrm{~K}$, suggesting possible structural change or pre-melting of GBs.

\subsection{Discussion}

In Fig. 2.5, the mobilities calculated by MD simulations are compared with those measured in previous experiments. In the experiments, the results are the average mobility for all GBs and they are measured at temperatures usually lower than those used in our MD simulations. Moreover, the experimental results may vary with samples by a couple of orders. At the same temperature range, the calculated results are found to be about four to five orders higher than experimental ones. This discrepancy is believed to be a result of impurity drag. In our simulations, we use stoichiometric $\mathrm{UO}_{2}$ without any impurities. However, in experiments the samples contained impurities at different levels. 


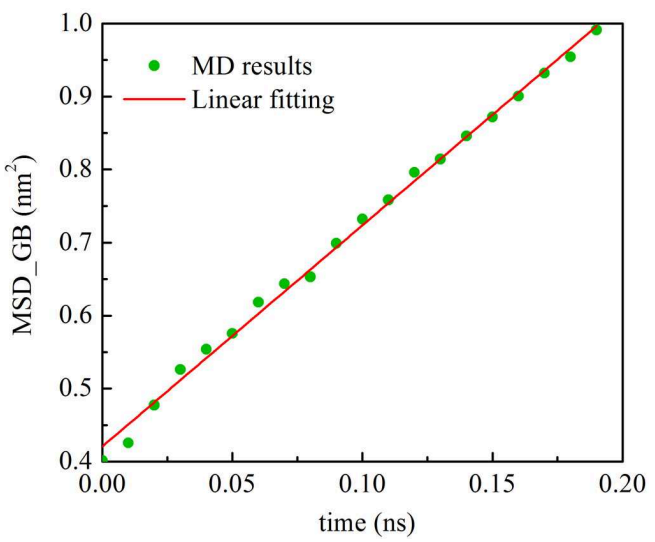

(a)

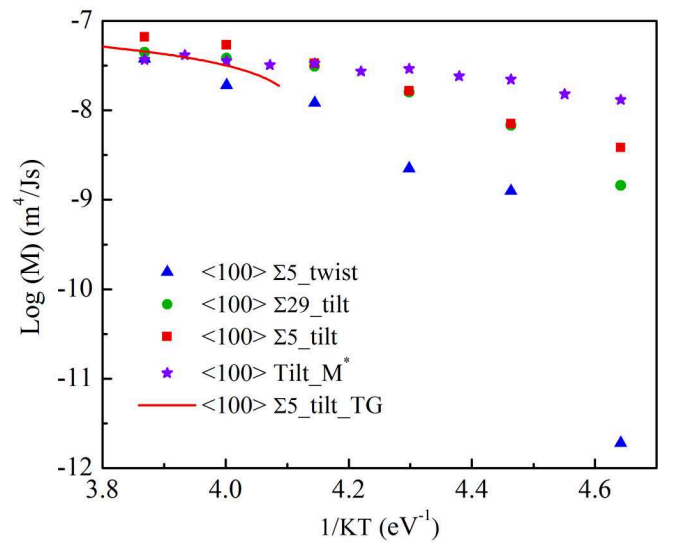

(b)

Figure 2.4: (a) MSD curve for the $\langle 100\rangle \Sigma 5\{310\}$ GB at $2700 \mathrm{~K}$ obtained with the randomwalk method, and (b) Logarithm mobilities of $\mathrm{UO}_{2}$ GBs obtained by various MD simulation methods. For the curvature-driving GB migration, a constant GB energy of $1.0 \mathrm{~J} / \mathrm{m}^{2}$ is used to convert the reduced mobility to mobility.

For instance, the concentration of Fe may reach the level of hundreds of APPMs in sintered $\mathrm{UO}_{2}$. Other impurities including $\mathrm{Al}$ and $\mathrm{Si}$ may present as well [2]. The existence of impurities, particularly those with strong segregation tendencies at GBs, may substantially reduce the GB mobilities from their intrinsic ones [14].

For impurities with non-zero segregation energies, enrichment (negative)) of depletion (positive) may occur at GBs at thermal equilibrium. During GB migration, the equilibrium segregation is disturbed and the impurities tend to remain in the GBs to a level as close to the thermal equilibrium as possible. For a certain GB with a constant migration velocity, a steady state segregation level will be established. The GB will drag the impurity load with it, and migrates at a velocity governed by the slowest moving species. This phenomenon is called impurity-drag.

In metals it has been shown that even in dilute alloy the impurity can significantly reduce the GB mobility [8]. For instance, in $\mathrm{Al}$ an impurity concentration of $10^{-6}$ can drop the mobility of the $\Sigma 7$ boundary by more than three orders [19]. In the literature, analytical models have been developed to explain the impurity drag [14], and the one based on the isothermal regular solution has been shown able to well match the experimental measurement [19]. This model has been numerically implemented. As shown in Fig. 2.6, both the GB impurity concentration $\left(\mathrm{C}_{b}\right)$ and the GB mobility $\left(\mathrm{M}_{b}\right)$ strongly depends on the global impurity concentration $\mathrm{C}$, with non-continuities at certain concentration. The key parameters required for this model are the segregation enthalpy and entropy, GB migration 


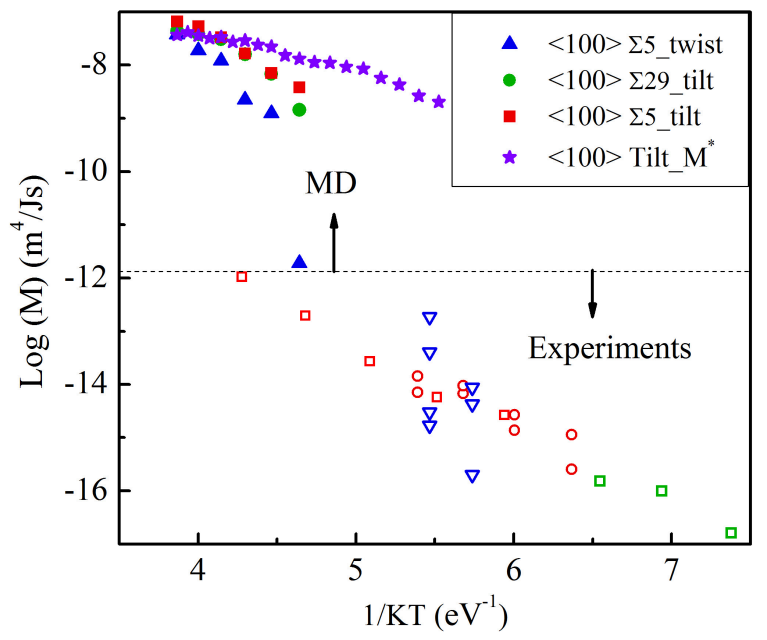

Figure 2.5: Logarithm mobility of $\mathrm{UO}_{2}$ GBs obtained from MD simulations and experiments.

barrier, and heat of mixing at GBs. Once the impurity specie is known, these parameters can be obtained from atomistic simulations to evaluate the impurity drag.

Given the large discrepancy between MD calculations and experiments, at this moment the experimental values are suggested to use in mesoscale simulations for the purpose to compare with experiments. By linear fitting the experimental data shown in Fig.2.5, a mobility is obtained as $M=2.75 * 10^{-6} \exp \left(-3.25 / K_{B} T\right)$. This value is the average from multiple experiments where the samples differ from each other in the senses of impurity level, grain size and porosities.

Though not directly comparable with experiments at this time, the MD results set the reference state for GB mobility in pure stoichiometric $\mathrm{UO}_{2}$. Without considering the impurity drag, these results can be taken as the upper bound of the GB mobilities. To be directly compared with experiments, several aspects need be clarified. First, the MD simulations suggest that there may be a transition in the migration barrier at about $2300 \mathrm{~K}$ due to probably the Bredig transition. As the experiments are usually done at lower temperature range, further simulations are needed to give the mobilities at the same temperature range as those in experiments. Second, the impurity drag effect needs be considered. It seems that the isothermal regular solution model is promising for this purpose, provided that the required parameters are available. Third, the experimental measurements give an average value to all GBs, while the MD simulations are usually for a specific GB, or a certain family of GBs. Thus, well-designed experiments are desired to validate the atomistic simulations. Once these effects are clarified, a more accurate description of GB mobility can be expected in $\mathrm{UO}_{2}$ fuels. 


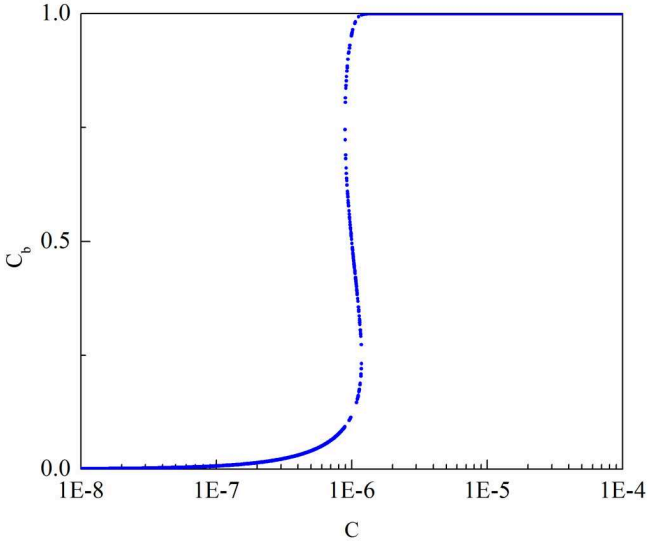

(a)

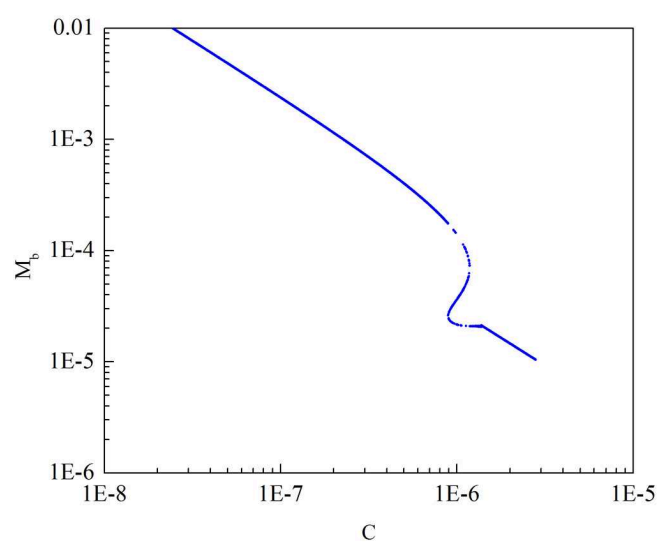

(b)

Figure 2.6: (a) Grain boundary impurity concentration and (b) mobility as functions of global impurity concentration. In the plotting the parameters used in Modolov et al. [19] have been used. 


\section{Chapter 3}

\section{Driving Force}

\subsection{Introduction}

GBs migrate in order to reduce the overall free energy of the system. Various driving forces can cause the GB motion. Possible driving forces include the drive to reduce the GB energy (the curvature driving force), the elastic energy, the defect energy, and a temperature gradient driving force. The curvature driving force is always present, including in $\mathrm{UO}_{2}$ fuel. While the elastic energy driving force is also present, it is typically small as elastic strains stay small due to plastic deformation and fracture. The defect energy driving force can be quite significant, however it is caused by gradients in the defect density which is fairly uniform in irradiated $\mathrm{UO}_{2}$. Thus, we neglect the elastic and defect energy driving forces for now. The temperature gradient driving force is also typically small, though fuel pellets experience very hight temperature gradients. Thus, it is possible that the temperature gradient driving force may be significant.

The magnitude of the curvature driving force is defined by

$$
P_{D F}=\frac{\sigma_{G B}}{R}
$$

where $\sigma_{G B}$ is the GB energy and $R$ is the GB curvature. The DF due to a temperature gradient $\nabla T$ can be expressed as $[8]$

$$
P_{D F}=\frac{\Delta S w_{G B}}{\Omega_{a}} \nabla T
$$

where $\Delta S$ is the entropy difference between the GB and the bulk, $w_{G B}$ is the GB width and $\Omega_{a}$ is the molar volume of the material. While this equation is presented in the text book, there are no references or derivation for the equation. 
In order to implement the driving forces in our average grain size model, there are two critical issues that must first be resolved. First, there are no GB energy values for $\mathrm{UO}_{2}$ that have been measured experimentally. Therefore, we must calculate it for various types of $\mathrm{UO}_{2}$ GBs using MD. Second, we must determine if the temperature gradient driving force equation from Gottstein and Shvindlerman [8] and identify if temperature gradient driving force is significant in light water reactor and fast reactor fuel.

\subsection{Grain Boundary Energy Calculation}

To calculate GB energies, we use molecular dynamics simulations with the LAMMPS [23] simulation package developed at Sandia National Laboratory. The Basak potential [5] which has been shown well reproduce the thermophysical properties of $\mathrm{UO}_{2}$ is chosen to describe the interatomic interaction in fluorite-phase $\mathrm{UO}_{2}$. To calculate the GB energy, a bicrystal model as shown in Fig. 3.1(a) is used. The top and bottom grains share the same $Y$ axes, and are rotated with respect to each other about the $Y$ axes so that a twist boundary is created. In each simulation cell there are two grain boundaries (one on top and the other in the middle) due to the periodicity applied along all three directions. The simulation cell dimensions vary with the misorientation angle. For each GB, the $\gamma$-surface mapping method [20] is used to search for the structure with the minimum formation energy. Once constructed, the simulation cells are firstly minimized to find the structure with the lowest energy, which is further quenched dynamically [40] to get the system energy at $0 \mathrm{~K}$. The quenching method sets the velocity of atom $i\left(\mathrm{v}_{i}\right)$ to zero if $f_{i} \cdot v_{i}<0$; here $f_{i}$ is the force acting on atom $i$. In the calculations the system pressure is kept zero using the NPH ensemble in LAMMPS.

Three families of symmetrical twist boundaries, with the rotation axes being $\langle 100\rangle,\langle 110\rangle$ and $\langle 111\rangle$ respectively, are used in the GB energy calculations. The results are shown in Fig. 3.1(b). For all three families of GBs, the energy increases initially with the misorientation angle, and then approaches a plateau at high-angle range. Assuming that the GBs are consist of series of dislocations, the GB energies in metals have been formulated theoretically using the Read-Shockley model [25], which was later modified by Wolf et al.[36] to have better description of high-angle GBs. In the model, the GB energy $\gamma_{G B}$ is given as a function of misorientation angle $\theta$ by:

$$
\gamma_{G B}=\sin (m \theta)\left(E_{c}-E_{s} \ln (\sin (m \theta))\right) / b
$$

In eq.3.3, $\mathrm{m}$ is the fold of symmetry, dependent on the rotation axis. $b$ is the Burgers vector of $\mathrm{GB}$ dislocations. $\mathrm{E}_{c}$ is the contribution of dislocation cores. $\mathrm{E}_{s}$ arises from the strain fields surrounding the dislocations and it is given as $E_{s}=\mu b / 2 \pi$ for twist GBs, and $E_{s}=\mu b / 4 \pi(1-\nu)$ for tilt ones. Here $\mu$ is the shear modulus; $\nu$ is the Poisson's ratio. As shown in Fig. 3.1(b), the MD results fit well with the theoretical model. In the fitting, we 


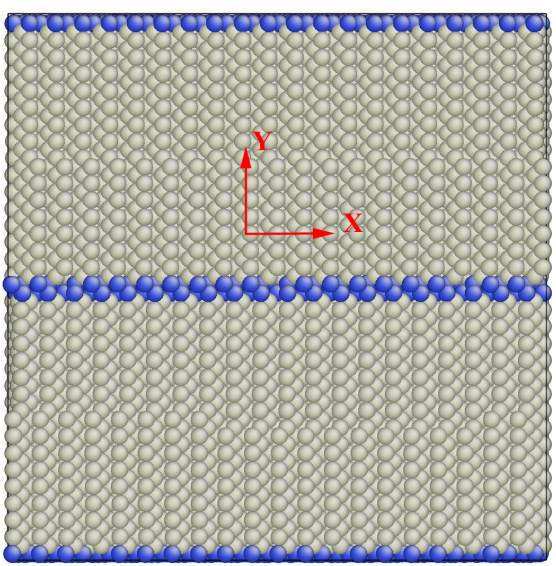

(a)

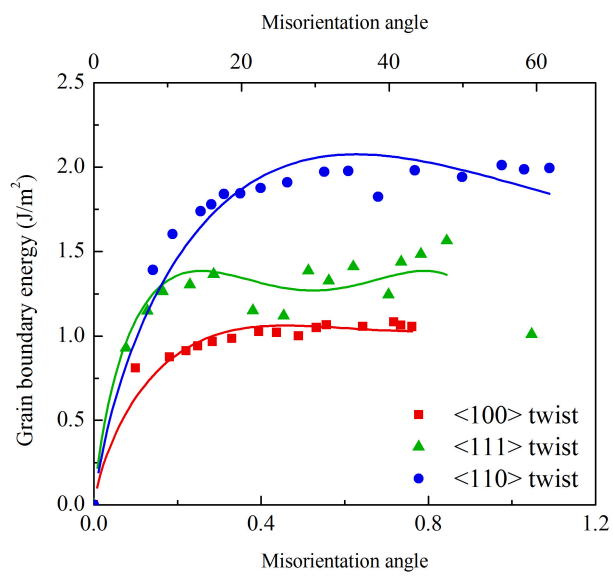

(b)

Figure 3.1: (a) Bicrystla model used for GB energy calculation and (b) GB energies as functions of misorientation angle. In (a) GB atoms are colored in blue and others in gray.

chose $b$ to the Burgers vector of full lattice dislocations, $1 / 2\langle 110\rangle$ for fluorite phase $\mathrm{UO}_{2}$. Other parameters obtained from the fitting are summarized in Table 3.1.

Table 3.1: Parameters used in the modified Read-Shockley model for $\mathrm{UO}_{2}$ twist boundaries.

\begin{tabular}{c|cccc}
\hline \hline Rotation axis & $m$ & $b(\AA)$ & $E_{c}\left(10^{-10} \mathrm{~J} / \mathrm{m}\right)$ & $E_{s}\left(10^{-10} \mathrm{~J} / \mathrm{m}\right)$ \\
\hline$\langle 100\rangle$ & 2 & 3.86 & 3.97 & 5.17 \\
\hline$\langle 110\rangle$ & 1 & 3.86 & 6.38 & 13.64 \\
\hline$\langle 111\rangle$ & 3 & 3.86 & 4.90 & 7.68
\end{tabular}

For the three families of twist GBs, the GB energies show some anisotropy regarding the GB characters. At high-angle range, the GB energy may vary by a factor of 2 by changing the rotation axis from $\langle 100\rangle$ to $\langle 110\rangle$. Also, it is interesting to notice that the $\langle 100\rangle$ twist GBs have the lowest energies, seconded by the $\langle 111\rangle$ GBs and then the $\langle 110\rangle$ GBs. This is in difference to the situation in face-centered-cubic (fcc) metals, for which $\langle 111\rangle$ twist boundaries are of the lowest energies. In the fluorite phase, the U-ion sublattice adopt the 
fcc structure and some similarity in GB energies with fcc metals is expected. However, in the fluorite structure each $\{111\}$ plane has three sub-layers, with the cation sub-layer sandwiched by two anion sub-layers. For grain boundaries with $\{111\}$ twist axis, two anion sub-layers will direct face each other with improper stacking and they repel each other due to the same type of charge. As a result, for $\mathrm{UO}_{2}$ the $\langle 111\rangle$ twist boundaries do not represent low energy boundaries any more, in contrast to the situation in fcc metals [35]. For the same reason, even for high symmetry boundaries such as the $\Sigma 3$ twin (note that coherent $\Sigma 3$ twin can be constructed by $60^{\circ}$ twist about the $\{111\}$ axis), no cusp in formation energy is shown.

Here, we calculate the GB energies using primarily the Basak potential. As has been shown by our earlier work with multiple potentials, the GB energy in $\mathrm{UO}_{2}$ obtained from MD simulations may depend on the interatomic potential used. However, these potentials shown the same trend regarding the GB character dependence [40]. Currently, there has been no density-functional-theory (DFT) calculations or experimental measurements on the grain boundary energies in $\mathrm{UO}_{2}$. The present results are consistent with previous molecular dynamics simulations [20], where it was shown that the Basak potential correctly predicts the structure of certain GBs in reference to DFT calculations.

\subsection{Verification of the Temperature Gradient Driving Force Equation}

To test the validity of the thermal gradient driving force equation (Eq. (3.2)), molecular dynamics (MD) simulation method is used. The testing strategy is briefly described as follows. In $\mathrm{MD}$ simulations the migration behavior of flat $\mathrm{GBs}$ in $\mathrm{UO}_{2}$ under a strong thermal gradient is firstly investigated. From simulations, the GB migration velocity can be directly extracted. At the mean time, the thermal gradient driving force can be estimated using Eq. (3.2). According to Eq. (1.1) and ignoring the resistive force, the GB mobility can be obtained. Since GB mobility is an intrinsic property of each GB, different calculation methods using different driving forces for GB motion should give similar GB mobilities. Therefore, if the GB mobility extracted from the thermal gradient driven simulations agrees well with those calculated from other standard methods discussed in Chapter 2, then Eq. (3.2) is the right theoretical equation to describe the thermal gradient driving force. To ensure the results are robust and not specific to one GB, multiple GBs of different GB characters are investigated. After the analytical equation of the thermal gradient driving force is verified, it will be used in phase field modeling to study the thermal gradient driven grain growth behavior at long time and length scales, which is relevant to the fuel restructuring problem in reactors.

The MD simulation package, LAMMPS [23], is used to study the GB motion driven by a 
thermal gradient in $\mathrm{UO}_{2}$. The widely used Basak potential [? ] is used to describe the interaction between ions. To exclude curvature driving force on GB motion and focus on thermal gradient driving force alone, five flat coincidence site lattice (CSL, or $\Sigma$ ) GBs in $\mathrm{UO}_{2}$ are studied: $\langle 110\rangle \Sigma 3\{111\}$ coherent twin, $\langle 100\rangle \Sigma 5\{310\}$ symmetric tilt, $\langle 100\rangle \Sigma 5\{310\}$ twist, $\langle 100\rangle \Sigma 29\{520\}$ symmetric tilt, and $\langle 100\rangle \Sigma 25\{710\}$ symmetric tilt. Here they are simply called as $\Sigma 3$ twin, $\Sigma 5$ tilt, $\Sigma 5$ twist, $\Sigma 29$ tilt, and $\Sigma 25$ tilt, respectively. To some extent, these GBs represent the complex nature of boundaries in materials. For example, $\Sigma 3$ twin GB is usually a low-energy boundary; $\Sigma 5$ tilt GB has a relatively high frequency of presence among special GBs as determined experimentally in $\mathrm{UO}_{2}[?] ; \Sigma 5$ twist GB represents high-angle twist boundaries; $\Sigma 29$ tilt GB is usually considered as a boundary close to random GBs; $\Sigma 25$ tilt GB has a misorientation angle of $16.3^{\circ}$ around $\langle 100\rangle$, which is a transition boundary between low- and high-angle boundaries. The gamma surface of each GB at $0 \mathrm{~K}$ is mapped out using the molecular statics calculations. The minimumenergy GB structure in the gamma surface is used for studying GB motion. Since periodic boundary conditions are employed in all directions, there are two equivalent GBs in each simulation system.

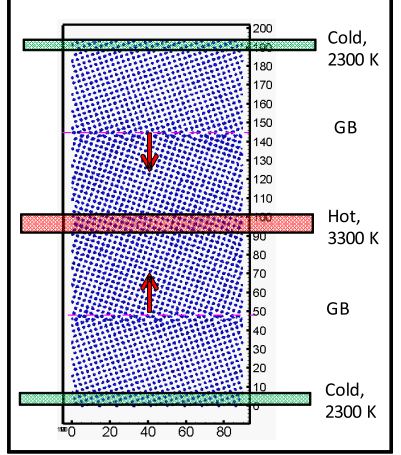

(a)

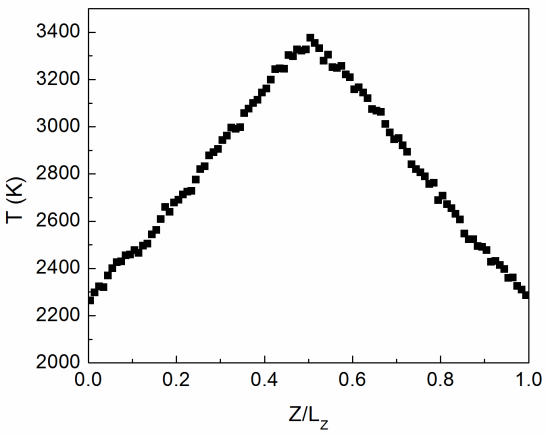

(b)

Figure 3.2: Illustration of creating a thermal gradient in a GB-containing system. (a) Typical simulation setup for creating a thermal gradient. (b) The temperature profile along $\mathrm{z}$ direction (same as the thermal gradient direction). $\mathrm{L}_{z}$ represents the total length of the simulation system in the $\mathrm{z}$ direction.

The simulation setup is shown in Fig. 3.2(a). In all simulations, a thermal gradient is imposed in the GB normal direction ( $\mathrm{z}$ direction), which has the longest length in each simulation system. The hot end is at the center, and the cold end is at both the top and bottom in $\mathrm{z}$ direction. The two GBs are at $0.25^{*} \mathrm{~L}_{z}$ and $0.75^{*} \mathrm{~L}_{z}$ respectively, where $\mathrm{L}_{z}$ is the length in the $z$ direction and typically about $200 \AA$ in all simulation systems. Since periodic boundary conditions are employed, the top and bottom regions belong to the same cold end. The thickness of thermostated region in the $\mathrm{z}$ direction is $10 \AA$ for both the hot 
and the cold end. The temperature is kept at $2300 \mathrm{~K}$ for the cold end and $3300 \mathrm{~K}$ for the hot end. Noting that the melting temperature (solid-liquid coexistence temperature) of $\mathrm{UO}_{2}$ predicted by the Basak potential is about $3540 \mathrm{~K}$ [9], slightly higher than the experimental value of $3147 \mathrm{~K}$ [13]. Therefore the material at the hot end still remains a crystalline structure in simulations. In all simulations, the time step is 2 fs. Before the simulation starts, the system volume is expanded to the equilibrium volume corresponding to the average temperature of the entire system $-2800 \mathrm{~K}$, and the entire system is relaxed at this temperature for $0.06 \mathrm{~ns}$. Then only the hot and cold ends are thermostated at their respective temperatures. Using this setup, two equivalent temperature gradients build up due to the applied periodic boundary conditions. An example of the temperature profile along the $\mathrm{z}$ direction is shown in Fig. 3.2(b). Typically the thermal gradient is about 100 $\mathrm{K} / \mathrm{nm}$, nearly 6 orders of magnitude higher than those built up in nuclear fuels. However, this high thermal gradient is necessary for observing GB motion at MD timescale.

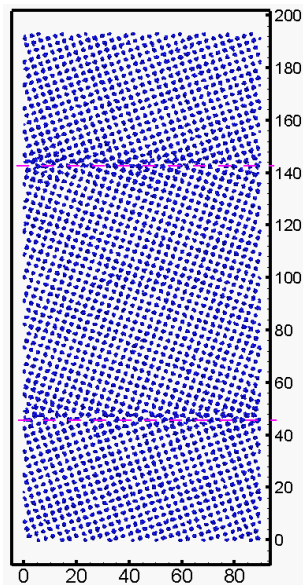

(a)

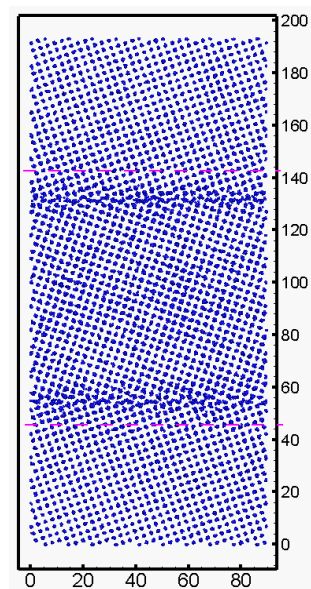

(b)

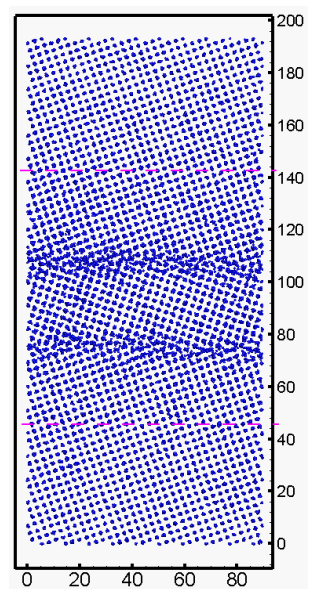

(c)

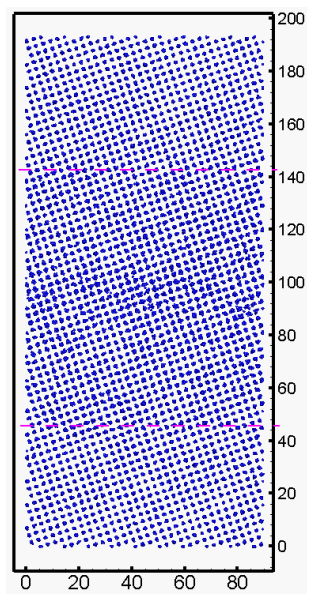

(d)

Figure 3.3: Four representative snapshots of GB positions at different simulation times for the migration of $\Sigma 5$ tilt GB under a thermal gradient. Only uranium atoms are shown for clarity. The dashed lines in each snapshot represent the initial positions of two GBs at $t$ $=0$ ns. (a) $\mathrm{t}=0.0 \mathrm{~ns}$; (b) $\mathrm{t}=0.8 \mathrm{~ns} ;$ (c) $\mathrm{t}=1.6 \mathrm{~ns}$; (d) $\mathrm{t}=1.8 \mathrm{~ns}$.

Figure 3.3 shows four representative snapshots of the migration of the $\Sigma 5$ tilt GB driven by a thermal gradient. Initially the two GBs are in the middle between the cold and hot ends (Fig. 3.3(a)) and their initial positions are indicated by two dash lines. Under the thermal gradient, the two GBs start to migrate towards the hot end. After the first time period of $0.8 \mathrm{~ns}$ (Fig. 3.3(b)), each GB migrates only about $7 \AA$ from their initial positions. After the second time period of 0.8 ns (Fig. 3.3(c)), each GB migrates substantially, about 30 $\sim 35 \AA$ from their respective previous positions in Fig. 3.3(b). The upper GB migrates a slightly more distance than the lower GB. After another $0.2 \mathrm{~ns}$ (Fig. 3.3(d)), the two GBs 
meet and anneal each other at the hot end and the system becomes a single crystal. During this shorter time of period, each GB migrates about $15 \AA$ from their respective previous positions in Fig. 3.3(c). The simulation clearly shows that the GB migration distance is not a linear function of time. In other words, GB migration velocity is not a constant. The GBs migrate faster and faster as they approach the hot end. This is because the local temperature at each GB keeps increasing as it migrates towards the hot end. Since GB mobility is an exponential function of temperature, its mobility and thus velocity keep increasing as well during the migration.

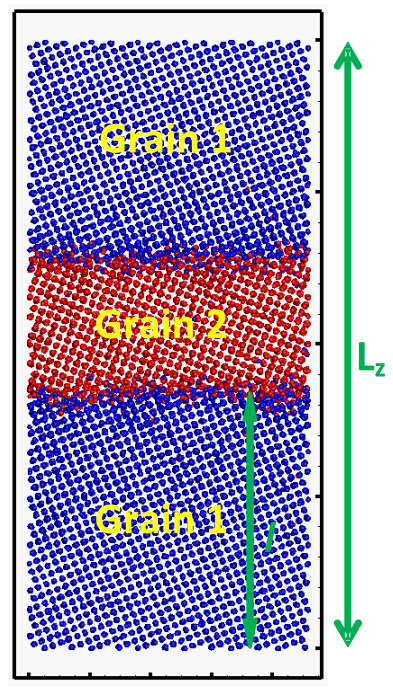

Figure 3.4: Characterization of different grains using orientation parameter analysis. Blue atoms belong to grain 1 and red atoms belong to grain 2 .

To calculate the GB migration velocities, an accurate method for tracking GB positions at different times is needed. As described in Chapter 2, the widely used orientation parameter analysis method [37] is employed to differentiate atoms belonging to different grains. Noting that only the uranium atoms are used for the analysis. Figure 3.4 shows the characterized GB positions by the orientation parameter analysis. It is clear that this method accurately determines the GB positions.

Using this method, the number of uranium atoms in grain $1\left(N_{1}\right)$ or in grain $2\left(N_{2}\right)$ can be obtained as a function of time. If $N_{1}$ is known, the length of the lower portion of the grain $1(l)$ shown in Fig. 3.4 can be estimated as,

$$
l=\frac{N_{1}}{N_{0}} \cdot \frac{L_{z}}{2}
$$

where $N_{0}\left(=N_{1}+N_{2}\right)$ is the total number of uranium atoms in the entire system, $L_{z}$ is the length of the simulation system in the GB migration direction (z direction). The average 
velocity of the two GBs is the time derivative of $l$,

$$
v=\frac{\mathrm{d} l}{\mathrm{~d} t}=\frac{\mathrm{d} N_{1}}{\mathrm{~d} t} \cdot \frac{L_{z}}{2 N_{0}} .
$$

Under a thermal gradient, the GB local temperature keeps changing as GBs migrate. Since GB mobility is an exponential function of temperature, the GB velocity also keeps changing as GBs migrate. The GB local temperature at a given GB position is

$$
T_{G B}=T_{l o w}+\frac{\mathrm{d} T}{\mathrm{~d} z} \cdot l=T_{l o w}+\frac{\left(T_{\text {high }}-T_{\text {low }}\right) \cdot N_{1}}{N_{0}},
$$

where $T_{\text {low }}$ and $T_{\text {high }}$ are the temperatures at the cold and hot ends respectively. Therefore, if $N_{1}$ at different simulation times is obtained, the GB migration velocity $(v)$ can be estimated using Eq. (3.5) and the instantaneous GB local temperature can be estimated using Eq. (3.6). Since the thermal gradient driving force $(F)$ can be estimated from the Eq. (3.2), the GB mobilities at different temperatures can be obtained using Eq. (1.1). These mobilities will be compared with the mobilities calculated from other established methods $[32,37]$ to test whether Eq. (3.2) describes the thermal gradient driving force correctly.

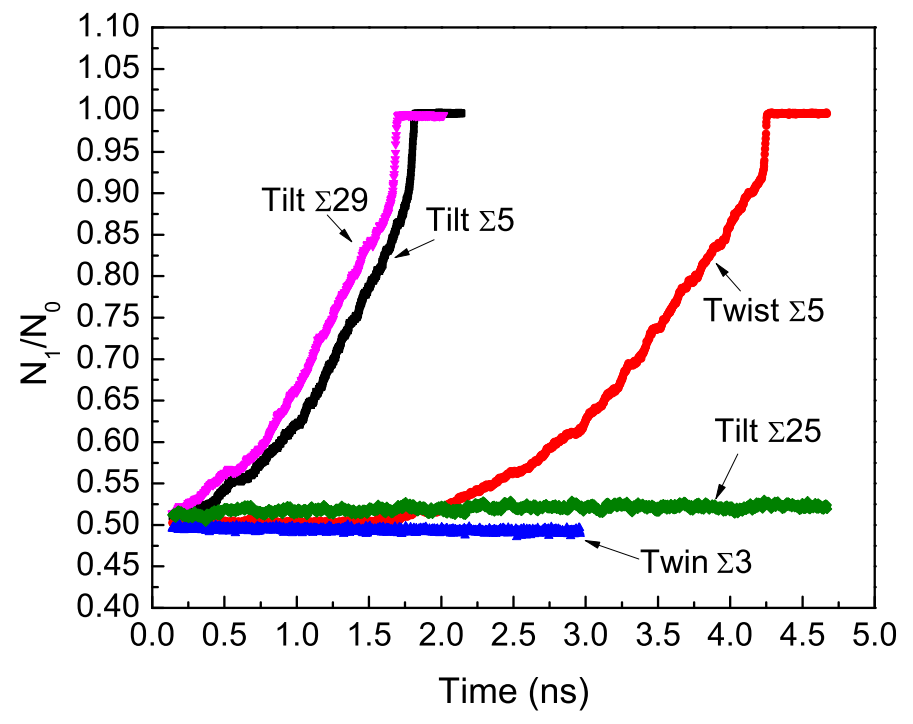

Figure 3.5: Comparison of the time-evolution of GB position (represented by the normalized number of atoms in grain 1) for five GBs under similar thermal gradients.

For the five GBs studied in this work, they have similar lengths in the GB migration direction so that the thermal gradient is similar in each system. Therefore, the rank of GB mobility can be qualitatively obtained by simply comparing their migration velocities. 
Figure 3.5 shows the normalized number of uranium atoms in the grain $1\left(N_{1}\right)$ as a function of time for the five GBs, where the normalization is relative to the total number of uranium atoms in their respective simulation systems. Since $N_{1}$ is related to the GB position (Eq. (3.4)), the slope of each curve is related to the GB migration velocity. Clearly the $\Sigma 29$ tilt and $\Sigma 5$ tilt GBs migrate very fast and the former migrates slightly faster, the $\Sigma 5$ twist GB migrates moderately, the $\Sigma 25$ tilt GB migrates very slowly, and the $\Sigma 3$ twin GB does not migrate at the MD timescale. This result clearly shows that GB mobility is GB-character-dependent. The mobility sequence is $\mathrm{M}_{\Sigma 3 \text { twin }}<\mathrm{M}_{\Sigma 25 \text { tilt }}<\mathrm{M}_{\Sigma 5 \text { twist }}<$ $\mathrm{M}_{\Sigma 5 \text { tilt }} \sim \mathrm{M}_{\Sigma 29}$ tilt.
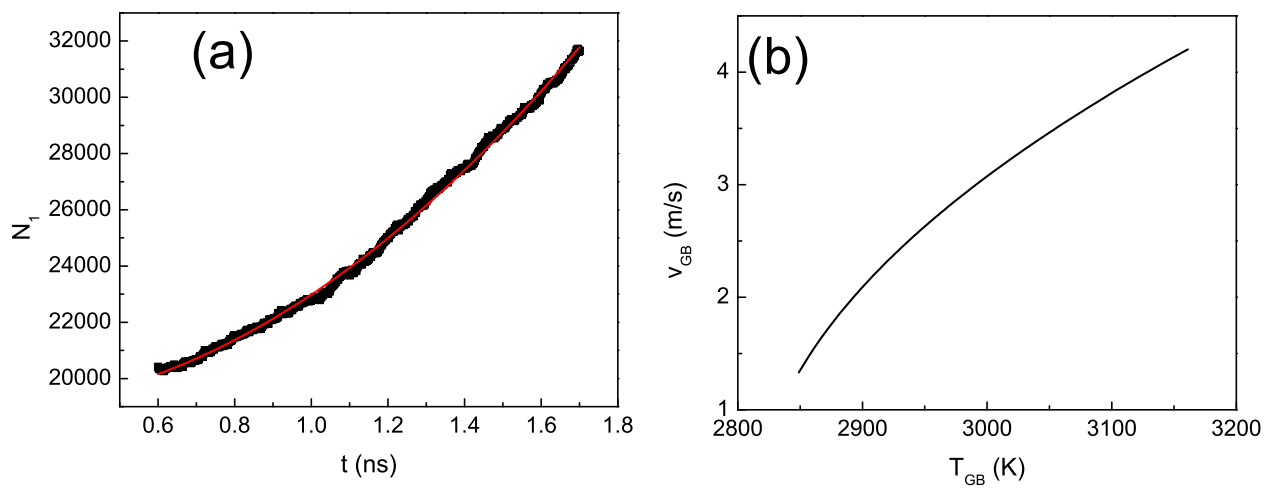

Figure 3.6: Extracting GB velocity from MD simulations for the $\Sigma 5$ tilt GB. (a) The 2nd order polynomial fitting of the number of uranium atoms in grain 1 with time. (b) The estimated GB migration velocity as a function of temperature.

Figure 3.5 only provides a qualitative comparison of GB mobility between different GBs. To quantitatively calculate the GB mobility, both the GB migration velocity and driving force are needed (Eq. (1.1)). Since the $\Sigma 3$ twin and $\Sigma 25$ tilt GBs either do not migrate or migrate a very small distance, they are excluded from the quantitative study of GB mobility. According to Eq. (3.5), the GB migration velocity is related to the time-derivative of the number of atoms in grain 1 . Since the GB migration velocity keeps changing as it migrates in a thermal gradient, one cannot simply use a linear fit of the data shown in Fig. 3.5 against time to extract the GB velocity for each GB. As an approximation, the number of atoms in grain 1 is fitted with a 2nd order polynomial function of time for each GB. An example of the polynomial fitting for the $\Sigma 5$ tilt GB is shown in Fig. 3.6(a). The fitting gives

$$
N_{1}=18922.8-940.6 \times t+5002.3 \times t^{2} .
$$

The time derivative of Eq. (3.7) gives

$$
\frac{\mathrm{d} N_{1}}{\mathrm{~d} t}=-940.6+1004.6 \times t
$$


Replacing $d N_{1} / d t$ in Eq. (3.5) with Eq. (3.8), the time-dependent GB velocity can be estimated. At a time $t$, the GB local temperature can be calculated using the combination of Eqs. (3.6) and (3.7). Therefore, the temperature-dependent GB velocity also can be obtained. An example is shown in Fig. 3.6(b).

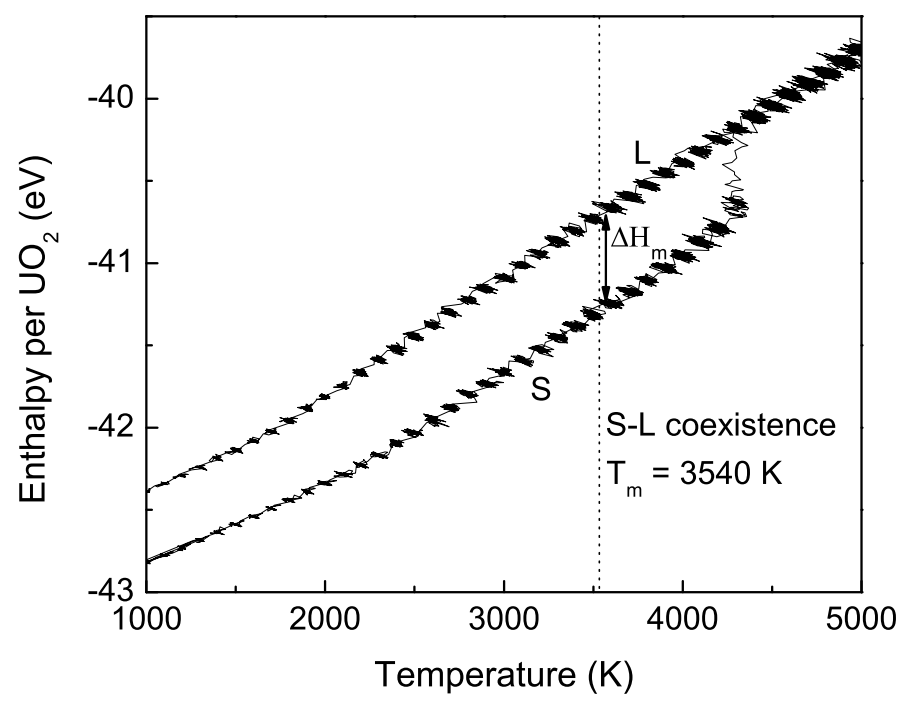

Figure 3.7: The enthalpy per $\mathrm{UO}_{2}$ molecule at different temperatures during a heatingcooling process in MD simulation.

For thermal gradient driving force (Eq. (3.2)), most of the parameters are well defined except for $\Delta S$ - the entropy difference between a single crystal and a GB. It may be very difficult to estimate this term accurately. However, if one assumes the extreme case of GB structure is a liquid-like amorphous structure, the upper limit of this entropy term is the entropy difference between a crystalline solid and a liquid, or the entropy of melting, $\Delta S_{m}$. To calculate the entropy of melting, a $\mathrm{UO}_{2}$ single crystal consisting of 6144 atoms is heated from $1000 \mathrm{~K}$ to $5000 \mathrm{~K}$ at zero external pressure to let it become a liquid. Then the liquid is cooled from $5000 \mathrm{~K}$ to $1000 \mathrm{~K}$. The heating/cooling rate is $5000 \mathrm{~K} / \mathrm{ns}$. The enthalpy per $\mathrm{UO}_{2}$ molecule at different temperatures during this heating and cooling process is shown in Fig. 3.7. The enthalpy increases sharply at about $4300 \mathrm{~K}$, indicating the crystal becomes a liquid at this temperature. However, this transition temperature is the superheating limit of the crystal because the surface melting is suppressed by the employment of periodic boundary conditions in MD simulation [4]. The true melting temperature $\left(T_{m}\right)$ is the solid-liquid coexistence temperature, which is usually much lower than the superheating limit. Govers et al. [9] calculated the solid-liquid coexistence temperature predicted by Basak potential, about $T_{m}=3540 \mathrm{~K}$. The enthalpy difference between the solid and liquid at this melting temperature is the latent heat of melting $\left(\Delta H_{m}\right)$, as shown in Fig. 3.7. Thus the entropy of melting for $\mathrm{UO}_{2}$ is 


$$
\Delta S_{m}=\frac{\Delta H_{m}}{T_{m}} \approx 15.5 \mathrm{~J} / \mathrm{mol} / \mathrm{K}
$$

This entropy difference is the upper limit of the entropy difference between a GB and a crystal. Interestingly, the authors of the book [8] specified $\Delta S=8 \times 10^{3} \mathrm{~J} / \mathrm{mol} / \mathrm{K}$. This value is about 3 orders of magnitude higher than that calculated from our MD simulation (Eq. (3.9)). In metals, the entropy of melting is close to the ideal gas constant, $R=8.31$ $\mathrm{J} / \mathrm{mol} / \mathrm{K}$ [24], a value close to our MD calculation. Therefore, the book must have a typo - the authors may mean $8 \mathrm{~J} / \mathrm{mol} / \mathrm{K}$ rather than $8000 \mathrm{~J} / \mathrm{mol} / \mathrm{K}$. If we use the wrong $\Delta S$, the calculated GB mobility would be about 3 orders of magnitude lower than it should be.

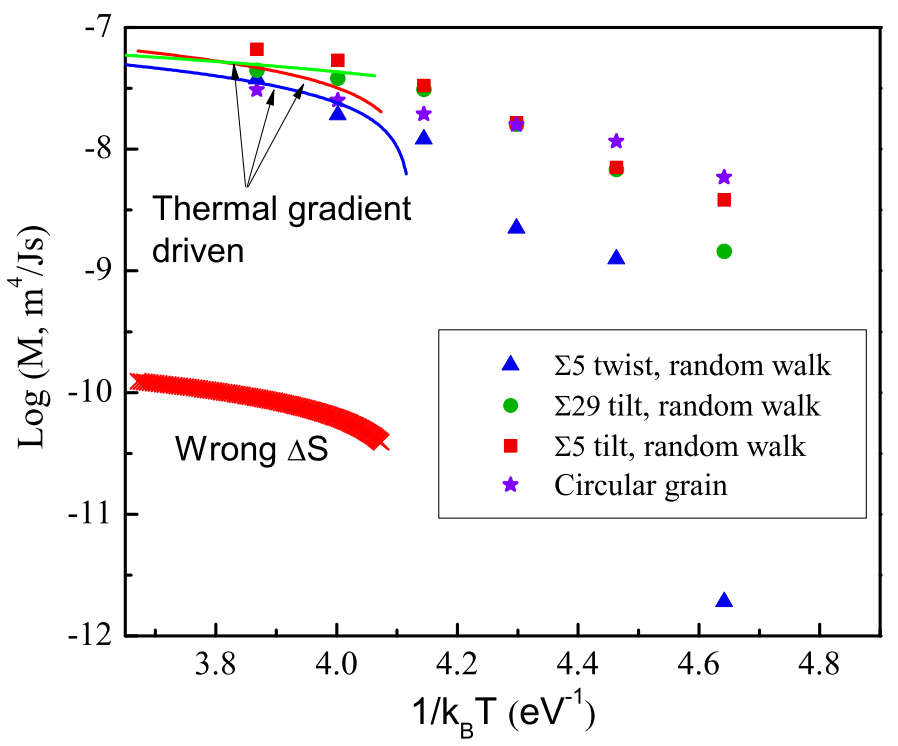

Figure 3.8: Comparison of GB mobilities calculated from three different methods in MD simulations (thermal gradient driven, curvature driven, and random walk).

After the temperature-dependent GB migration velocity and driving force are obtained, the temperature-dependent GB mobility is calculated by using Eq. (1.1), or M=v/F. The Arrhenius plots of the calculated GB mobility for three GBs are shown in Fig. 3.8. To calculate the thermal gradient driving force, the GB thickness is set to $w_{G B}=1 \mathrm{~nm}$; The molar volume of $\mathrm{UO}_{2}$ molecules is about $\Omega_{a}=2.44 \times 10^{-5} \mathrm{~m}^{3} / \mathrm{mol} ; \Delta S=15.5 \mathrm{~J} / \mathrm{mol} / \mathrm{K}$; and the thermal gradient in the simulation depends on the respective length in the $\mathrm{z}$ direction, typically about $\mathrm{dT} / \mathrm{dz}=100 \mathrm{~K} / \mathrm{nm}$. For comparison, the wrong value of $\Delta S=8 \times 10^{3}$ $\mathrm{J} / \mathrm{mol} / \mathrm{K}$ provided in the book [8] is also used and the resulting GB mobility for the $\Sigma 5$ tilt GB is also shown in Fig. 3.8. 
To further test the thermal gradient driving force, the GB mobilities calculated in Chapter 2 using the two well-established methods (curvature driven and random walk) are also shown in Fig. 3.8. Since GB mobility is an intrinsic property for a GB, different methods should give a similar value for the same GB [15] - at least the difference should not be more than a few orders of magnitude. For the random-walk method, the calculated GB mobilities of the three CSL GBs have the order, $\mathrm{M}_{\Sigma 5 \text { twist }}<\mathrm{M}_{\Sigma 5 \text { tilt }} \sim \mathrm{M}_{\Sigma 29 \text { tilt }}$, which agrees well with the mobility sequence obtained in the thermal gradient driven method. For the curvature-driven method, the calculated mobilities are close to those of the two high-mobility GBs ( $\Sigma 5$ tilt and $\Sigma 29$ tilt), suggesting that these two high-mobility GBs are similar to random GBs. For the thermal gradient driven method, both the wrong entropy value provided in book and the correct entropy value calculated from MD are used to estimate the GB mobility. Clearly, if the wrong $\Delta S$ is used, the estimated GB mobility is about 3 orders of magnitude lower than those calculated from the two standard methods. On the other hand, if the MD-calculated $\Delta S_{m}$ is used, the GB mobility agrees very well with those calculated from the two standard methods. This good agreement is consistent with the fact that GB mobility is an intrinsic property and different methods should give similar values for the same GB. Since the thermal gradient driving force is used as input for calculating GB mobility, the good agreement also verifies that the thermal gradient driving force equation (Eq. (3.2)) is valid, as long as a correct $\Delta S$ is used in this equation. Noting that the MD-calculated $\Delta S_{m}$ is the upper limit of $\Delta S$. If the true $\Delta S$ is a half of $\Delta S_{m}$, the calculated mobilities from the thermal gradient driven method would only shift upwards slightly in the logarithm plot of Fig. 3.8 and the agreement between different methods would still be good.

In this work, the GB migration behavior driven by a thermal gradient in $\mathrm{UO}_{2}$ is investigated using MD simulations. Five representative GBs of different GB character are studied. The qualitative comparison shows that their mobilities follow the order: $\mathrm{M}_{\Sigma 3 \text { twin }}<\mathrm{M}_{\Sigma 25 \text { tilt }}$ $<\mathrm{M}_{\Sigma 5 \text { twist }}<\mathrm{M}_{\Sigma 5 \text { tilt }} \sim \mathrm{M}_{\Sigma 29}$ tilt. The GB mobility does not correlate strongly with GB energy, misorientation angle, tilt/twist, CSL $\Sigma$ number, suggesting that GB mobility is a complex material property and it is not a simple function of any single GB character.

The major goal of this work is to test the validity of the thermal gradient driving force equation proposed previously. Using the GB migration velocity obtained from MD simulations and the theoretical thermal gradient driving force equation, the mobilities of three GBs are calculated quantitatively. The obtained GB mobilities are compared with those calculated from two well-established methods and good agreement between three methods is obtained. The good agreement demonstrates that the previously proposed theoretical equation of the thermal gradient driving force is valid, except that the entropy term is about three orders of magnitude larger than it should be (possibly due to a typo in the book). The validated theoretical equation of the thermal gradient driving force can then be used in mesoscale modeling such as phase field method [31] to study the GB migration behavior under a thermal gradient. In reality, many different driving forces coexist so that 


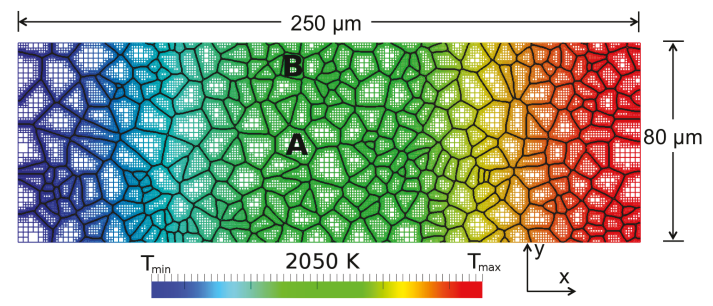

(a)

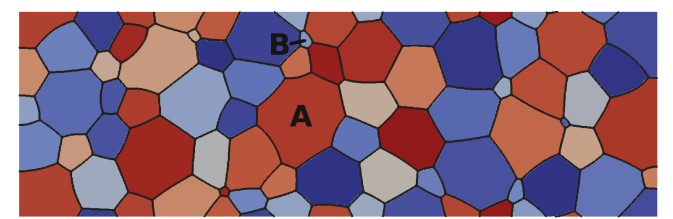

(b)

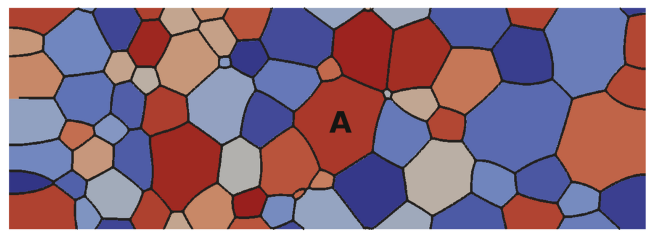

(c)

Figure 3.9: Polycrystal simulation domain, where the initial grain configuration showing the adapted mesh is shown in (a). The constant temperature gradient is also shown. Note that the values for $\mathrm{T}_{\min }$ and $\mathrm{T}_{\max }$ vary for the different gradients, ranging from $\mathrm{T}_{\min }=$ $\mathrm{T}_{\max }=2050 \mathrm{~K}$ for no gradient to $\mathrm{T}_{\min }=1950 \mathrm{~K}$ and $\mathrm{T}_{\max }=2150 \mathrm{~K}$ for $\nabla T=0.8$ $\mathrm{K} / \mu \mathrm{m}$. The centroid position with time is recorded for the grains labeled A and $\mathrm{B}$. The final grain configuration after 2000 minutes is shown in (b) with no temperature gradient and in (c) with a gradient of $0.8 \mathrm{~K} / \mu \mathrm{m}$, where grain B has disappeared. Note that with the temperature gradient, the grains are smaller on the cold side and larger on the hot side.

understanding their relative importance at long time and length scales is important. This task has been conducted using phase field modeling and the results are presented in the next section.

\subsection{Investigation of Significance of Temperature Gradient Driving Force}

In this section we determine if the temperature gradient driving force is significant using a series of 2D phase field grain growth simulations using the NEAMS MARMOT tool. We summarize the work here, focusing on the results. We detail the approach and verify the model in Tonks et al. [31].

We investigate the importance of the temperature gradient driving force (DF) with $\mathrm{UO}_{2}$ fuel. We model a $\mathrm{UO}_{2}$ rectangular $2 \mathrm{D}$ domain that is $250 \mu \mathrm{m}$ by $80 \mu \mathrm{m}$ with 300 initial 
grains. The grains were initialized using a Voronoi tessellation and have an initial average grain size of $8.2 \mu \mathrm{m}$, with symmetric boundary conditions (BCs) for the PF model on the left and right and periodic BCs on the top and bottom. The grain growth is modeled for 2000 minutes. We apply five linear temperature gradients, all with an average temperature of $2050 \mathrm{~K}$. The area of each grain is recorded with time, such that we can calculate the distribution and mean of the grain size. The simulations employed the same parameter values as the bicrystal simulations from the previous section. Mesh adaptivity was employed to decrease the computational expense. See Fig. 3.9(a) for the initial polycrystal domain with the adapted mesh.

All the simulations, irrespective of the temperature gradient, reduced from the initial 300 grains to $67 \pm 2$ grains after 2000 minutes. The final microstructures with no temperature gradient and with $\nabla T=0.8 \mathrm{~K} / \mu \mathrm{m}$ are shown in Figs. 3.9(b) and 3.9(c), respectively. By comparing the position of grain A (shown in Figs. 3.9(a) and 3.9(c)), it is clear that there is significant grain migration towards the hot end of the polycrystal in the presence of a temperature gradient. To obtain a quantitative measure of the grain migration velocity, we fit a line to the $x$-position of the centroid of grains A and B (Figs. 3.9(b)) at four different times for the five different temperature gradients, showing a linear migration towards the hot side of the fuel, demonstrated for grain A in Fig. 3.10(a).

To investigate these grain velocities, we compare them to the GB velocity calculated from Eqs. (1.1) and (3.2) at $\mathrm{T}=2050 \mathrm{~K}$, which represents the maximum possible grain velocity at that temperature. The actual grain velocity at the same average temperature will always be less than this ideal GB velocity, as it depends on the orientation of its GBs with respect to the temperature gradient. As shown if Fig. 3.10(b), the grain velocity increases with increasing temperature gradient, but not linearly as with the ideal velocity. In addition, the grain velocity appears to be independent of grain size, as grains A and B show similar behavior even though grain A is significantly larger than grain B.

For grain growth due to the curvature DF, the average grain size changes linearly with time, where the slope is a function of the DF, Eq. (3.1). However, this behavior is only obtained once the grain size distribution has reached a steady-state form. In our simulation with a zero temperature gradient, the grain area reaches this equilibrium behavior after 1000 minutes, and the slope $\dot{A}=\sigma_{G B} M$, as shown in Fig. 3.11(a). The behavior is consistent with the curvature DF. In addition, all the simulations, irrespective of the temperature gradient, exhibit a similar linear increase with the same slope.

We also plotted the size of the largest grain with time and found some impact of the magnitude of the temperature gradient on its behavior. The change in the size of the largest grain is accelerated after 1000 hours for the 0.6 and $0.8 \mathrm{~K} / \mu \mathrm{m}$ gradients (see Fig. 3.11(b)), with the acceleration increasing with temperature gradient. The final grain size distribution (Fig. 3.11(c)) shows that this acceleration in the change of the largest grain size with time is due to the formation of a slightly bimodal grain size distribution, with a slight peak at 


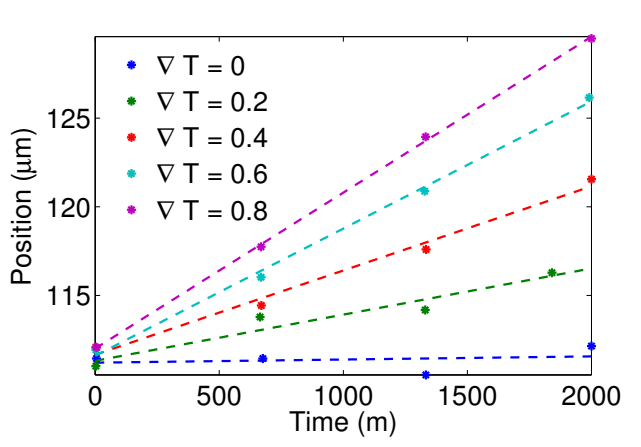

(a)

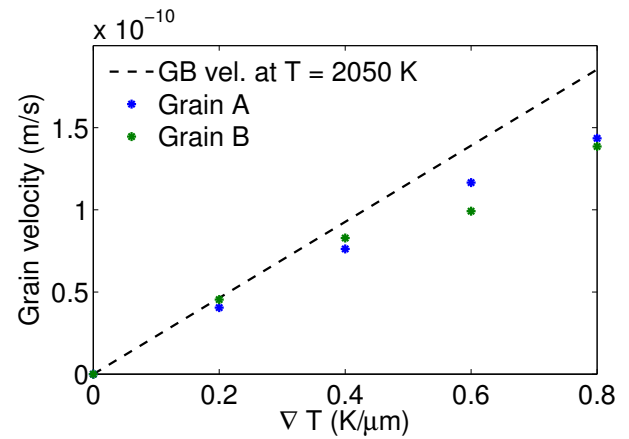

(b)

Figure 3.10: Velocity of grains A and B, where (a) shows how the grain velocity is calculated (shown with values from grain A) from the slope of linear fits to the x-position of the grain centroid for each temperature gradient, where the fits are shown as dashed lines. (b) shows the grain velocity of A and B as a function of the temperature gradient. The GB velocity predicted by Eqs. (1.1) and (3.2) at $\mathrm{T}=2050 \mathrm{~K}$, which represents the maximum possible GB velocity at that temperature, is shown as a black dashed line.

the largest grain sizes.

To elucidate the cause of this change in the grain size distribution, we compare approximate magnitudes of the curvature and temperature gradient DFs. We approximate the curvature DF using Eq. (3.1), with the radius of curvature calculated from the average grain area $A_{a v}$ according to $R=\sqrt{A_{a v}} / 2$. The temperature gradient DF is approximated using Eq. (3.2). However, note that this approximation of the temperature gradient DF represents a maximum value (only experienced by GBs perpendicular to the temperature gradient), while our approximation of the curvature DF represents an average value. As shown in Fig. 3.11(d), the average curvature DF decreases with time, due to the increase in average grain area, while the temperature gradient DF is constant. For all grain sizes in our simulations, the average curvature DF is greater than the maximum temperature gradient DF, even if the temperature gradient DF was increased by $9.5 \%$ to account for the error observed in our bicrystal simulations, indicating why the temperature gradient did not significantly impact the change in the average grain area (Fig. 3.11(a)). However, independent of the DF, the GB mobility will always be higher in hotter regions of a polycrystal. Thus, grains on the hot end grow larger than those on the cold end, causing the observed change in the grain size distribution. This gradient in grain size is clearly observable in the final microstructure from the $0.8 \mathrm{~K} / \mu \mathrm{m}$ gradient shown in Fig. 3.9(c). Note that if we normalized each grain size by the average grain size at the corresponding temperature, the grain size distribution would be unchanged by the temperature gradient, as shown in Garcia et al. [6]. 
The average temperature gradient experienced by the fuel in LWRs is about $0.2 \mathrm{~K} / \mu \mathrm{m}$, while it is about $0.4 \mathrm{~K} / \mu \mathrm{m}$ in oxide fuel fast reactors. Thus, from our simulations it appears that the temperature gradient DF will have no impact on the average grain size within the fuel. In fact, the maximum temperature gradient DF will be smaller than the average curvature DF for all fuels with an average grain size less than $97.0 \mathrm{~mm}$ for LWR conditions and less than $48.5 \mathrm{~mm}$ for fast reactor conditions (with the DFs calculated as before). However, the temperature dependence of the GB mobility will have an effect, resulting in faster moving GBs and thus larger grains in the hotter portion of the fuel.

While the temperature gradient has little impact on the average grain boundary behavior, it will have an impact on the local behavior. Our simulations showed a distinct motion of grain A for all temperature gradients greater than zero (Fig. 3.10(b)). This grain motion within the fuel caused by the temperature gradient could result in additional fission gas pick-up, increased interaction between fission gas bubbles and GBs, and local modifications of the stress fields due to grain anisotropy.

The temperature gradient DF could also play an important role in non-nuclear applications. Local grain migration will take place in any application with a significant temperature gradient and could impact the local microstructure behavior. In applications with a significant temperature gradient and a large grain size, the curvature DF will be reduced and the temperature gradient DF may play a more dominant role than observed in our simulations. By equating the DFs from Eqs. (3.1) and (3.2), we can solve for the critical grain size

$$
d_{c r i t}=\frac{\Delta S w_{G B}}{2 \sigma_{G B} \Omega_{a}} \nabla T,
$$

where the average grain size $d=2 R$. Thus, for given polycrystal parameters and temperature gradient, the temperature gradient DF may play a dominant role if the average grain size is greater than $d_{c r i t}$. Again note that this approximation of the temperature gradient DF represents a maximum value while the approximation of the curvature DF represents an average value.

GBs are driven to migrate up temperature gradients, adding an additional GB migration DF. Here, we have implemented the temperature gradient DF into a PF model of grain growth. We verified the model by comparing to the analytical equation (3.2) and found that the PF model predicts GB velocities that are $9.5 \pm 0.5 \%$ slower than those calculated by the analytical model. The phase field model was then used to predict the behavior in a $\mathrm{UO}_{2}$ polycrystal with a range of temperature gradients. We found that while the temperature gradient does cause local grain boundary motion up the gradient, the evolution of the average grain area with time is not impacted. This is because the temperature gradient DF is smaller than the curvature DF for all of the gradients that we investigated. The behavior of the largest grain size with time does change with the gradient, due to changes in the grain size distribution. However, these changes are not caused by the temperature 
gradient DF, but rather by the dependence of the GB mobility on temperature. Therefore, the temperature gradient DF may not need to be considered in macroscale models of the average grain size in LWR and fast reactor fuel, though the temperature gradient will modify the local grain behavior. 


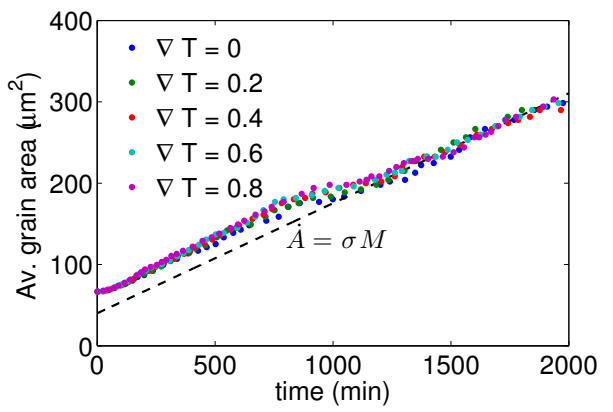

(a)

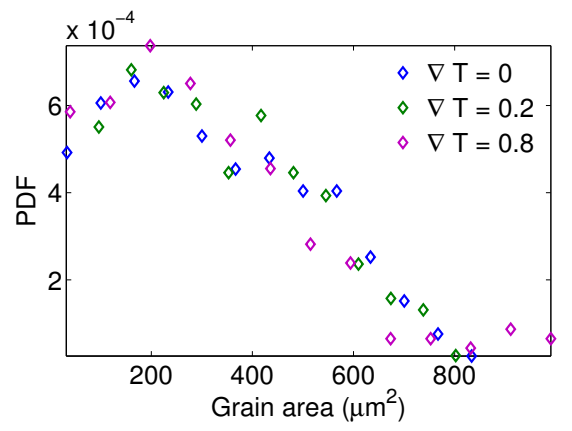

(c)

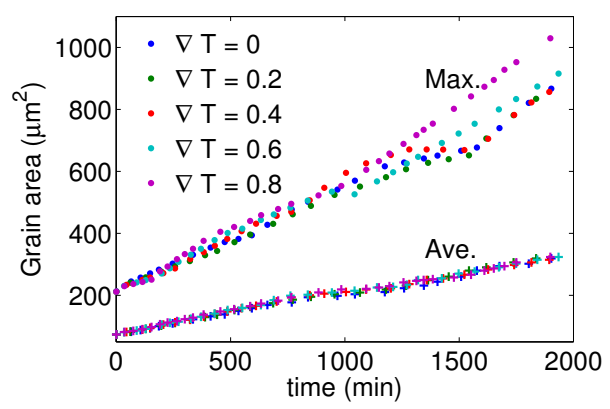

(b)

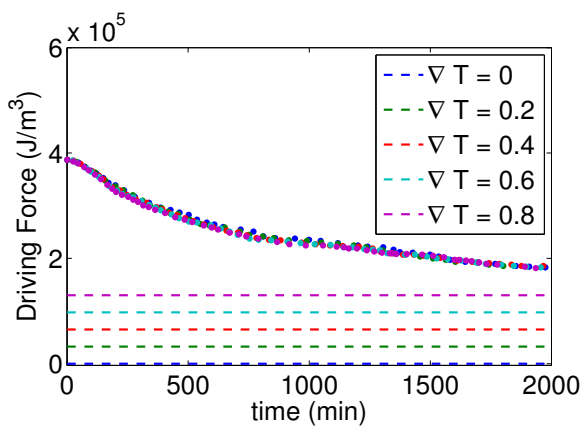

(d)

Figure 3.11: Quantified measures of the polycrystal grain growth, where all temperature gradient units are $\mathrm{K} / \mu \mathrm{m}$. In (a), the average grain area is plotted with time, with a linear slope $\dot{A}=\sigma M$ after enough time for the grain structure to adjust from the initial Voronoi tessellation. Note that the average grain area does not change with temperature gradient. However, the behavior of the maximum grain size with time does change for the larger two gradients, as shown in (b), where the dots indicate the maximum grain area and the plus signs the average. A numerical approximation of the probability density function (PDF) of the final grain size distribution for three of the temperature gradients is shown in (c), where it is evident that for the largest gradient, a slight bimodal distribution has formed, with an increase in grains of large size. In (d), the curvature DF (dots) and the temperature gradient DF (dashed lines) are compared, clearly showing that the temperature gradient $\mathrm{DF}$ is smaller than the curvature DF for all gradients used in our simulations. 


\section{Chapter 4}

\section{Resistive Force}

Various defects present in irradiated $\mathrm{UO}_{2}$ fuel resist GB motion, where the most dominant are pores (voids and bubbles). Two main distributions of pores exist in the material, voids left over from the sintering process used to fabricate the pellets and fission gas bubbles that form on GBs during irradiation. To describe the combined pinning force from these two types of pores requires an analytical model.

The earliest treatment of GB pinning was by Zener [26]. In Zener's original model, he considered spherical, incoherent, and immobile particles on migrating GBs. He determined the pinning force to be equal to

$$
F_{Z}=2 \pi r \sigma_{G B} \cos \beta \sin \beta,
$$

where $r$ is the particle radius, $\sigma_{G B}$ is the GB energy, and $\beta$ the angle illustrated in Fig. 4.1. He determined that the maximum force occurs at $\beta=45^{\circ}$, such that

$$
F_{z}^{\max }=\pi r \sigma_{G B}
$$

This model can be applied for particles but also for pores and bubbles. Similar expressions have been derived using an energy argument that the particle takes the place of some GB area, reducing the GB energy [3].

To determine the pinning pressure, Zener made simplifying assumptions; he assumed that the GBs were flat, the particles were randomly distributed, only particles within one radius influence the GBs, and that all particles exert the maximum pinning force throughout the interaction. With these assumptions, Zener determined that the pinning pressure per unit area of GB is equal to

$$
P_{Z}=\frac{3 f_{v} \sigma_{G B}}{4 r},
$$




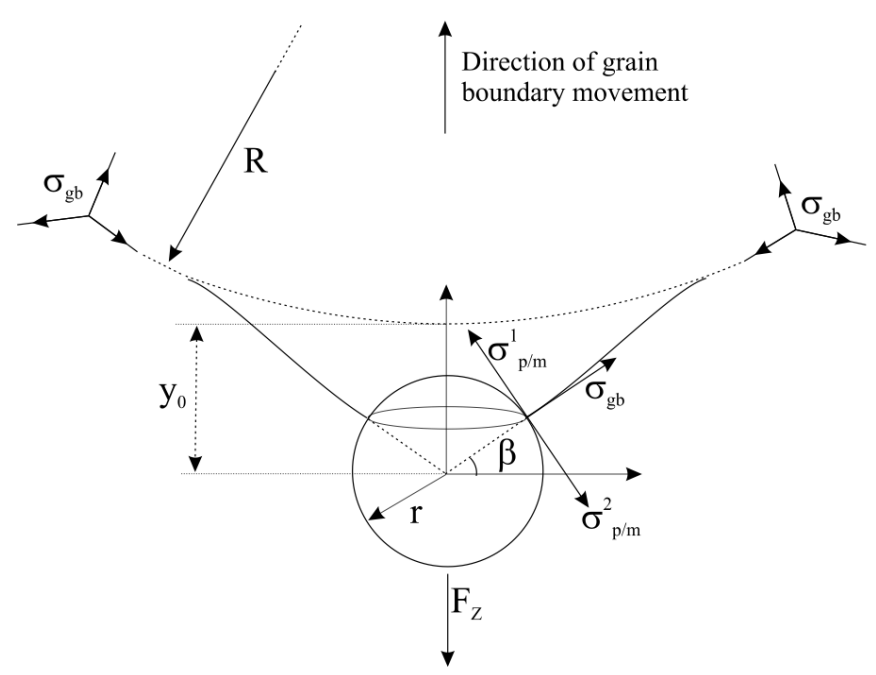

Figure 4.1: Interaction between a spherical particle and a grain boundary that meets the particle at an angle $\beta$, where the particle radius is $r$ (figure is taken from Moelans et al. $[17])$.

where $f_{v}$ is the volume fraction of particles. The assumptions that the GBs are flat is not consistent with actual GB behavior, as GB's form a catenoid-like shape around a pinning object. In addition, as particles pin GBs, a larger fraction are located on the GBs then indicated by the assumption of random positioning of the GB with respect to the particles. Thus, Zener's model has been modified by later work to remove these assumptions and improve the model predictions [7, 3, 10, 27, 11].

Both the sintering porosity and fission gas bubbles do not fit the assumptions made by Zener, and thus cannot be accurately described by Zener's model. While a model exists in the literature that describes the resistive force of sintered porosity, no model exists for GB porosity, such as fission gas bubbles, defined by the GB fractional coverage (the percentage of the GB covered by particles). The fractional coverage is calculated by the fission gas release model and is how the grain size model will couple to that model. In the following sections we first summarize the pinning model for sintered porosity and validate it by comparing to experimental data. Second, we develop a pinning model that describes the pinning pressure of GB bubbles in terms of the GB fractional coverage. 


\subsection{Sintered Porosity Model}

The model presented in [12] was developed to describe the pinning force for sintered porosity using

$$
P_{r}=N_{s} \frac{f_{v} \sigma_{G B}}{r_{b}}
$$

where $N_{s}$ is a stereographic parameter that quantifies the amount of GB-particle contact beyond random. In an earlier work [22], the value for $N_{s}$ for various materials was measured, including for $\mathrm{UO}_{2}$ for which $N_{s}=2.8$. To ensure that this model accurately predicts the resistive force of sintered pores in $\mathrm{UO}_{2}$, we compare to data from Ainscough et al. [2].

In that work, measurements of grain growth rates were made on twelve types of stoichiometric $\mathrm{UO}_{2}$ specimens, mainly at temperatures between 1573 to $1773 \mathrm{~K}$. Initial pellet densities varied from $94 \%$ to $99 \%$ theoretical. We have used Eq. (4.4) to predict the grain growth on a subset of the same samples, considering the initial porosity, average grain size, and the temperature. The average pore radius, a critical parameter in the model, was not reported therefore were determined the average pore radius by fitting to the data. Some densification was reported in the paper, and we used the density changes that they measured.

We calculated the change in grain size using Eq. (1.2), considering only the curvature driving force (Eq. (3.1)). Thus, the final model used for the comparison is:

$$
\dot{D}=2 M \sigma_{G B}\left(\frac{2}{D}-2.8 \frac{f_{v}}{r_{b}}\right)
$$

We use the GB mobility that was fitted from the data in Ainscough et al. [2] and the GB energy from Nerikar et al. [20].

The model compares fairly well with the data, as shown in Figure 4.4, though there are some points that deviate from the model. These appear to be due to uncertainty in the data, as the grain size decreases with time in some cases, which would not actually occur during sintering.

\subsection{GB Bubble Pinning Model}

Zener based his pinning pressure on the volume fraction of particles $f_{v}$, as this is an observable and measurable quantity to allow for validation of the model and facilitate its applicability in real systems. Here, we derive an expression for the pinning pressure in terms of the GB fractional coverage. We begin with the pinning force, from Eq. (4.1), and 


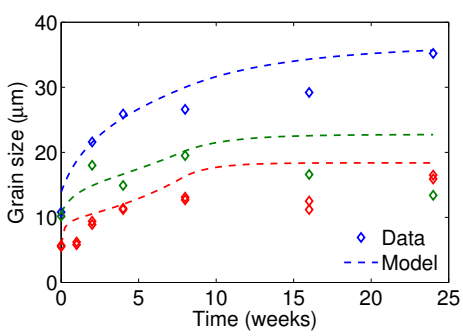

(a)

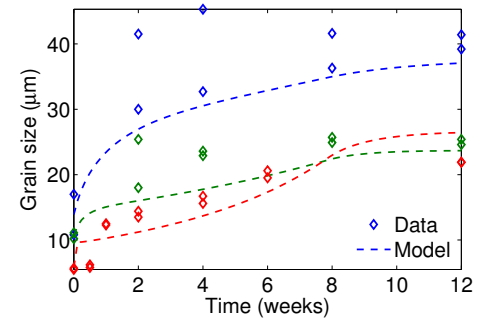

(b)

Figure 4.2: Comparison of the analytical pinning model for sintered porosity from Eq. (4.4) to grain growth data from $\mathrm{UO}_{2}$ from Ainscough et al. [2], where the colors represent data from different batches, with different density and initial grain radius. The points show the experimental data and the lines are the analytical model.

multiply by the the number of bubbles per unit area in contact with the GB to get the pinning pressure

$$
P_{G B}=2 \pi r \sigma_{G B} \cos \beta \sin \beta \frac{N_{b}}{A_{G B}},
$$

where $N_{b}$ is the total number of GB particles and $A_{G B}$ is the total GB area. While this expression is does not make any assumptions about the positioning of particles with respect to the GB, it has limited applicability as it is extremely difficult if not impossible to measure $N_{b}$ and $A_{G B}$. This situation can be somewhat improved by employing the GB fractional coverage $f_{c}=N_{b} A_{b} / A_{G B}$, or the percentage of the GB covered by particles, i.e.

$$
P_{G B}=2 \pi r \sigma_{G B} \cos \beta \sin \beta \frac{f_{c}}{A_{b}},
$$

where $A_{b}$ is the cross section of a single particle crossing the GB. We simplify this expression by assuming a circular particle cross section and flat GB such that $A_{b}=\pi r^{2}$. Thus, the pinning pressure is

$$
P_{G B}=\frac{2 \sigma_{G B} f_{c} \cos \beta \sin \beta}{r},
$$

and the max pinning pressure would occur at $\beta=45^{\circ}$ with a value of

$$
P_{G B}^{\max }=\frac{\sigma_{G B} f_{c}}{r} .
$$

As the GB migrates passed the pinning particles, it does not stay flat but rather takes on a catenoid shape, as shown in Fig. 4.1. To account for the catenoid shape, Zener's model 
was modified with the exponent $a=0.93$ [10], thus increasing the pinning pressure. To account for the catenoid shape in our model, we include a weighting term $W$ to give

$$
\begin{aligned}
P_{G B} & =2 W \frac{\sigma_{G B} f_{c} \cos \beta \sin \beta}{r} \\
P_{G B}^{\max } & =W \frac{\sigma_{G B} f_{c}}{r} .
\end{aligned}
$$

To verify that this analytical model accurately describes the pinning pressure, and to determine a value for $W$, we run a series of phase field simulations of GB migration with pinned boundaries.

\subsubsection{Pore/GB Interaction Phase Field Model}

To model the interaction between GBs and pinning particles, we model the GB migration in Mo with He bubbles. We employ a simple phase field model based on that presented in Millett and Tonks [16]. However, we add the directional model of bulk, surface, and GB diffusion from Ahmed et al. [1]. In the model, grains are represented by order parameters $\eta_{i}$ equal to one within a corresponding grain and equal to zero in the other grains, assuming isotropic GB properties. The voids are represented with a conserved vacancy concentration $c$ that is equal to one within a void and some small concentration within the bulk. The variables evolve to minimize the total free energy in the system, representing the microstructure evolution. The free energy of the system is defined in terms of the model variables as

$$
\begin{aligned}
F= & \int_{V}\left(\mu f\left(c, \eta_{i}\right)+\frac{\kappa_{c}}{2}|\nabla c|^{2}+\right. \\
& \left.\sum_{i} \frac{\kappa_{i}}{2}\left|\nabla \eta_{i}\right|^{2}\right) d V
\end{aligned}
$$

where $\kappa_{c}$ is the interfacial parameter for $c, \kappa_{i}$ is the interfacial parameter for the $i$ th order parameter and $\mu$ is the bulk energy parameter. The bulk energy term is defined as

$$
\begin{aligned}
f\left(c, \eta_{i}\right)= & \sum_{i}\left(\frac{\eta_{i}^{4}}{4}-\frac{\eta_{i}^{2}}{2}\right)+\left(\frac{c^{4}}{4}-\frac{c^{2}}{2}\right)+ \\
& \gamma_{G B} \sum_{i} \sum_{j>i} \eta_{i}^{2} \eta_{j}^{2}+\gamma_{c} \sum_{i} c^{2} \eta_{i}^{2},
\end{aligned}
$$

where $\gamma_{G B}$ is typically set to 1.5 to ensure a symmetric diffuse interface [18] and $\gamma_{c}$ is defined by the surface energy to GB energy ratio. 
The evolution of the concentration $c$ is defined by a Cahn-Hilliard equation according to

$$
\frac{\partial c}{\partial t}=\nabla \cdot \mathbf{M} \nabla \frac{\partial F}{\partial c(\mathbf{r}, t)}
$$

where $\mathbf{M}$ is the concentration mobility matrix that accounts for directional diffusion along surfaces and GBs. The evolution of the order parameters $\eta_{i}$ is defined by an Allen-Cahn equation, as

$$
\frac{\partial \eta_{i}}{\partial t}=-L \frac{\partial F}{\partial \eta_{i}}
$$

where $L$ is the order parameter mobility and for this model it is assumed to be equal for all GBs.

The model has a number of parameters that must be determined as functions of measurable quantities in order to make a material specific model. These quantities are the GB energy $\sigma_{G B}$, surface energy $\sigma_{s}$, vacancy or gas bulk vacancy diffusivity $D_{b u l k}$, and the GB mobility $M_{G B}$. For the order parameter values, we use the model presented in Moelans et al. [18], which gives the relationships

$$
\begin{aligned}
\kappa_{i} & =\frac{3}{4} \sigma_{G B} l_{G B} \\
L & =\frac{4}{3} \frac{M_{G B}}{l_{G B}} \\
\mu & =6 \frac{\sigma_{G B}}{l_{G B}} .
\end{aligned}
$$

where $l_{w}$ is the interfacial width that defines the width of the diffuse interfaces in the phase field model. The mobility can be determined by the fact that the concentration evolution should follow Fick's law of diffusion for $c \ll 1$ removed from a GB, such that

$$
\mathbf{M}=\mathbf{D} / \frac{\partial^{2} f}{\partial c^{2}}=\mathbf{D} /\left(2 \mu\left(\gamma_{c}-1\right)\right)
$$

The parameter $\gamma_{c}$ is set by the ratio of the surface energy to the GB energy, i.e.

$$
\gamma_{c}=\gamma_{G B} \frac{\sigma_{s}}{\sigma_{G B}}=\frac{3}{2} \frac{\sigma_{s}}{\sigma_{G B}} .
$$

The concentration $c$ impacts the free energy in the same manner as $\eta_{i}$, therefore

$$
\kappa_{c}=\frac{3}{4} \sigma_{s} l_{G B} .
$$


The gas diffusivity matrix defines the total diffusivity due to bulk, surface and GB diffusion following the model from Ahmed et al. [1]. The diffusivity matrix is equal to

$$
\mathbf{D}=D_{b u l k}\left(\hat{\mathbf{D}}_{b}+\hat{\mathbf{D}}_{s}+\hat{\mathbf{D}}_{G B}\right)
$$

where the bulk diffusivity

$$
D_{b u l k}=D_{0} e^{-\frac{E m}{k_{b} T}}
$$

with the diffusivity prefactor $D_{0}$, formation energy $E_{m}$, the Boltzman constant $k_{b}$, and the temperature $T$. The individual normalized diffusivity tensors are determined as

$$
\begin{aligned}
\hat{\mathbf{D}}_{b} & =w_{b} \mathbf{I} \\
\hat{\mathbf{D}}_{s} & =w_{s} c^{2}\left(1-c^{2}\right) \mathbf{T}_{s} \\
\hat{\mathbf{D}}_{G B} & =w_{G B} \sum_{i=1} \sum_{j \neq i} \eta_{i} \eta_{j} \mathbf{T}_{G B}
\end{aligned}
$$

where the surface projection tensor is a function of the concentration according to

$$
T_{s}=\mathbf{I}-\frac{\nabla c}{|\nabla c|} \otimes \frac{\nabla c}{|\nabla c|}
$$

The GB projection tensor is a function of the order parameters as

$$
T_{G B}=\mathbf{I}-\frac{\nabla \eta_{i}-\nabla \eta_{j}}{\left|\nabla \eta_{i}-\nabla \eta_{j}\right|} \otimes \frac{\nabla \eta_{i}-\nabla \eta_{j}}{\left|\nabla \eta_{i}-\nabla \eta_{j}\right|}
$$

The phase field equations are solved using the finite element method in the MARMOT mesoscale modeling code [28]. The order parameters are solved in the manner outlined in that paper. The concentration is solved by splitting the fourth order Cahn-Hilliard equation into two second order equations in the manner summarized in Zhang et al. [39]. All of the equations are solved simultaneously using implicit time integration.

To model GB and void interaction the Mo-He system, we require values for the GB mobility and energy in Mo. We also need the He diffusivity in Mo (in terms of prefactor $D_{0}$, the migration energy $E_{m}$, the Mo surface energy $\sigma_{s}$. We obtain these values from molecular dynamics (MD) simulations conducted using the potential developed in ]. The values are shown in Table 4.1.

We verify that our phase field model is accurately capturing the pinning behavior by modeling a circular grain of Mo with ten bubbles randomly distributed along the GB using $\mathrm{MD}$ and the phase field model. The $3 \mathrm{D}$ domain is $64.4 \mathrm{~nm} \times 64.4 \mathrm{~nm} \times 1.93 \mathrm{~nm}$ with periodic boundary conditions. A circular grain with radius of $20 \mathrm{~nm}$ is embedded in a 
Table 4.1: Materials parameters for Mo with He bubbles, calculated using MD.

\begin{tabular}{|l|l|}
\hline Property & Value \\
\hline GB energy & $2.4 \mathrm{eV}$ \\
$\mathrm{M}_{G B}^{0}$ & $3.986 \times 10^{-6} \mathrm{~m}^{4} / \mathrm{J} \mathrm{s}$ \\
$\mathrm{Q}$ & $1.0307 \mathrm{eV}$ \\
$\mathrm{D}_{H e}^{0}$ & $3.1376 \times 10^{-7} \mathrm{~m}^{2} / \mathrm{s}$ \\
$\mathrm{E}_{m}^{H e}$ & $0.94498 \mathrm{eV}$ \\
\hline
\end{tabular}

larger grain and ten $0.3 \mathrm{~nm}$ radius He bubbles are randomly located along the circular GB. The circular grain will decrease in size, but the GB motion is inhibited by the He bubbles, as shown in Fig. 4.3(a). The decrease in the volume of the circular grain with time is predicted by MD and by the phase field model, and the results are compared. Multiple simulations are run using both approaches, with different random positions of the ten $\mathrm{He}$ bubbles.

Both approaches predict an initially slow decrease in the grain volume (see Fig. 4.3(b)). However, the rate increases as the GB releases from more and more of the ten bubbles until the final rate of decrease is defined by the GB mobility, as no bubbles are in contact with the GB. The shrinkage of the circular bubble predicted by the two methods has a similar rate when all ten bubbles are in contact with the GB (before $1 \mathrm{~ns}$ ). However, after this time, the phase field model predicts a faster rate of decrease than the MD simulations. The final rate of decrease of the volume predicted by the two approaches, once all bubbles are released, is identical. The difference between the two approaches appears to be that the MD simulations predict that the bubbles migrate along with the GB for some time, slowing their eventual release from the GB. The phase field model does not predict this migration. However, as the goal of this work is to investigate GB pinning and not the dragging of pores, we are comfortable with the performance of the phase field model.

As a final test of the phase field model, we compare the simulation results against the analytical model from Eq. (4.6). The number of bubbles in contact with the GB is recorded from the simulation and used in the equation. As the GB slides past the ten He bubbles over time, the $\beta$ contact angle changes with time. Therefore, we employ an average contact angle of $22.5^{\circ}$. The phase field model simulations compare well with the model predictions, as shown in Fig. 4.3(c).

\subsubsection{Investigation of the Analytical Model}

To verify the analytical model from Eq (4.10), we employ the phase field model presented in the previous section. We again look at Mo bicrystals with He bubbles, but we employ a bicrystal with dimensions $0.2 \mu \mathrm{m} \times 0.4 \mu \mathrm{m} \times 0.4 \mu \mathrm{m}$, with a flat GB with an artificial 


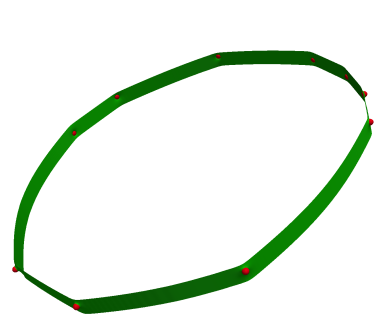

(a)

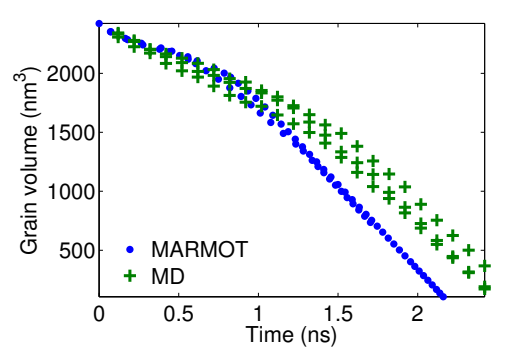

(b)

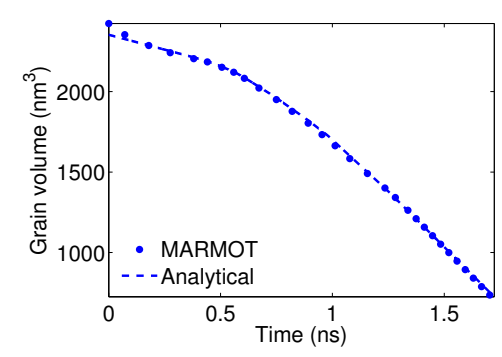

(c)

Figure 4.3: 2D

driving force moving along the x-direction, as shown in Fig. 4.4(a). The GB width is $w_{G B}=3.1 \mathrm{~nm}$. Increasing numbers of bubbles are lined up on a plane parallel to the GB plane in the path of the migrating GB. Simulations are conducted with bubbles of radius $7 \mathrm{~nm}, 9 \mathrm{~nm}$, and $11 \mathrm{~nm}$. The artificial driving force is added to the model by modifying Eq. (4.14) (similar to the temperature gradient driving force from Tonks et al. [31]) according to

$$
\frac{\partial \eta_{i}}{\partial t}=-L \frac{\partial F}{\partial \eta_{i}}+A \mathbf{f}_{d} \cdot \nabla \eta_{i}
$$

where $A=4 /\left(3 l_{G B}\right)$ with the interfacial with of the model $l_{G B}=3.1 \mathrm{~nm}$ and the driving force $\mathbf{f}_{d}=98.1 \hat{\mathbf{i}} \mathrm{MPa}$.

A plot of the grain volume vs time for various numbers of $9 \mathrm{~nm}$ radius bubbles is shown in Fig. 4.4(b). Note that change in volume of the grain is linear until the GB comes in contact with the bubbles. At this time, the rate increases as the GB is pulled towards the bubbles. This is consistent with the pinning force equation (Eq. (4.1)) for values of $\beta$ less then zero. As the GB moves pass the bubbles, the rate of change of the volume decreases until the GB reaches an angle of $\beta=45^{\circ}$, where the pinning force is maximum. The volume changes at a fairly constant rate at this point of maximum pinning force for some time, until bubbles begin to release. Eventually, the GB releases from all GBs and its rate returns to that before it impacted the bubbles. Though all the curves follow this same trend, the changes in the velocity increase with increasing numbers of bubbles.

To simplify the comparison between our phase field results and the analytical equation defining the pinning pressure as a function of the fractional coverage, we focus on the time at which the contact angle $\beta$ is near $45^{\circ}$, when the pinning force is a maximum. To determine the GB velocity during this period, we fit a line to the phase field data and use the slope as the velocity. From this velocity, we calculate the pinning pressure from Eqs. (1.1) and (4.10). 


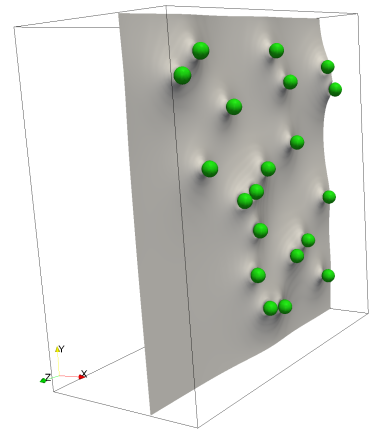

(a)

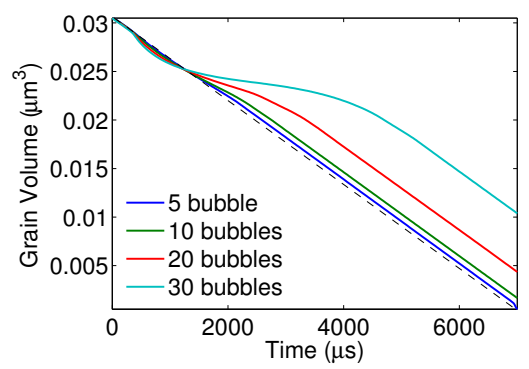

(b)

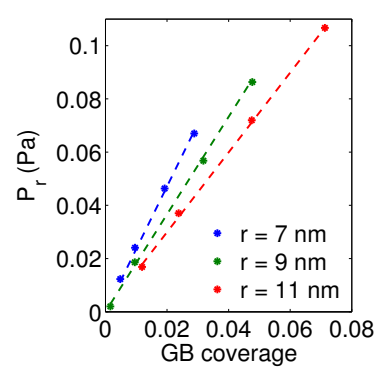

(c)

Figure 4.4: 2D

With a coefficient of $W=1.1$ to represent the catenoid shape of the pinned GB, the maximum pinning pressure predicted by the analytical model (Eq. (4.10)) compares very closely with that predicted by the phase field method. Figure 4.4(c) shows the pinning pressure vs the GB fractional coverage. The phase field model clearly shows a linear relationship between $P_{G B}$ and $f_{c}$, as shown by the analytical model. In addition, the pinning pressure varies inversely with the bubble radius. Thus, by comparing with the phase field results, we have identified the proper value for $W$ and verified that the analytical model correctly predicts the material behavior. 


\section{Chapter 5}

\section{Bison Implementation}

To predict the average grain size throughout the fuel as a function of temperature and porosity, we use the analytical model

$$
\dot{D}=2 M_{G B}\left(P_{D F}-P_{r}\right) .
$$

The GB mobility $M_{G B}$ has been calculated using MD simulations, as summarized in Chapter 2 as a function of temperature and impurities. We have considered two possible driving forces that contribute to $P_{D F}$, the curvature driving force and the temperature driving force. In Chapter 3 we use phase field simulations to show that the temperature gradient driving force is negligible. Finally, in Chapter 4 we determine that the resistive force due to the porosity left over from sintering and that from the fission gas bubbles on the GBs must be treated differently. Thus, we have developed two separate models. In this Chapter, we discuss how the model will be implemented in BISON and what work still needs to be completed for the model to be finished.

As mentioned in the previous paragraph, only the curvature driving force was found to be significant in reactor fuel. Therefore, the driving force can be approximated as

$$
P_{D F} \approx \frac{2 \sigma_{G B}}{D} .
$$

The resistive force due to sintered porosity and fission gas bubbles is

$$
P_{r}=2.8 \frac{\sigma_{G B} f_{V}}{r_{s}}+1.1 \frac{\sigma_{G B} f_{c}}{r_{f g}}
$$

where $f_{V}$ is the volume fraction of sintered porosity, $r_{s}$ is the average radius of the sintered

porosity, $f_{c}$ is the GB fractional coverage, and $r_{f g}$ is the average radius of the GB fission gas bubbles. 


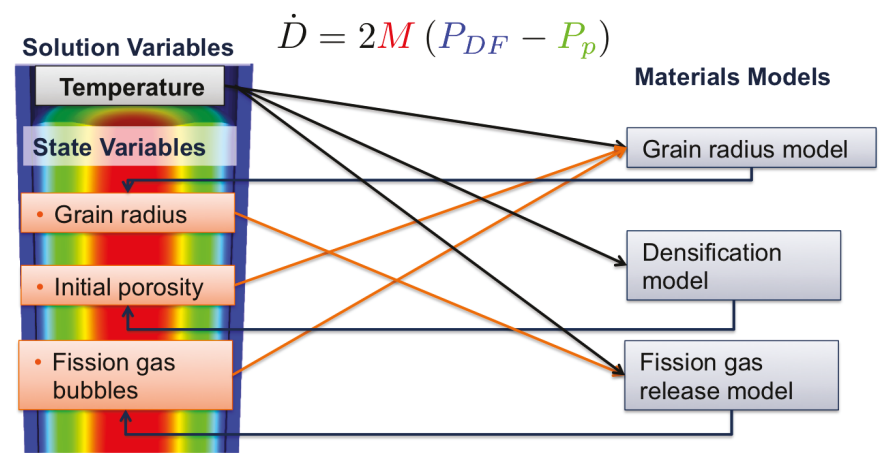

Figure 5.1: Illustration of the implementation of the grain growth model in BISON, showing its interaction with the fission gas release and densification models.

This model has been implemented in the BISON code, in a similar manner to the existing emperical model from Ainscough et al. [2]. However, the model is a function of four additional microstructure variables, the volume fraction and average radius of sintered porosity, $f_{V}$ and $r_{s}$, respectively, the GB fractional coverage $f_{c}$, and the average radius of the fission gas bubbles on the GBs $r_{f g}$. The sintered porosity volume fraction and average radius should be evolved in the densification model in BISON. The current densification model in BISON is emperical and only evolves the volume fraction, so we currently assume a constant average pore radius. Next year we will develop a mechanistic sintering model which will replace the emperical one and will evovle both $f_{V}$ and $r_{s}$. The fractional coverage and the average radius of GB bubbles are both currently evolved by the fission gas release model. Thus, our grain growth model is a function of temperature, and interacts with both the densification and fission gas release models, as shown in Fig. 5.1.

While the basic structure of the model is in place and implemented in BISON, it is not yet complete. The work that still needs to be completed is:

- Complete calculation of GB mobility in $\mathrm{UO}_{2}$, taking into account impurity drag.

- Account for differences in fission gas bubble density on different GB types.

- Investigate the impact of bubble dragging on GB motion. An additional model may be needed to account for drag of bubbles once the GB is completely pinned.

- Completion of BISON assessment cases using the new grain growth model, to verify its impact on the BISON calculations.

This work should be completed by end of this fiscal year. 


\section{Chapter 6}

\section{Conclusion}

The next generation fuel performance capability is under development as part of the NEAMS Fuels Product Line. The macroscale BISON code provides the advanced modeling capability, however new materials models that are based on microstructure rather than burnup are required (see Fig. 1.1). We are developing these models using the multiscale approach illustrated in Fig. (1.2).

In this report, we have summarized our development of a mechanistic material model

to predict the evolution of the average grain size in $\mathrm{UO}_{2}$ fuel. The model follows the form

$$
\dot{D}=2 M_{G B}\left(P_{D F}-P_{r}\right)
$$

where the GB mobility $M_{G B}$ is determined using MD simulations, the driving force $P_{D F}$ is developed using a combination of MD simulations and mesoscale phase field simulations using INL's MARMOT code. The resistive force caused by sintered pores was taken from the literature, but validated against $\mathrm{UO}_{2}$ data. The resistive force caused by fission gas bubbles forming on the GBs was defined by a new analytical model that was derived here as a function of the fission gas GB fractional coverage. The model was informed by MARMOT simulations of GB motion in the presence of aligned GBs. Current work is focusing on understanding the impact of GB anisotropy of the average grain size and quantifying the importance of bubble dragging. The completed model will be implemented in BISON, and coupled to the fission gas release and sintering models. A number of BISON assessment cases will be rerun using the new model to quantify its impact. 


\section{Bibliography}

[1] K. Ahmed, C. Yablinsky, A. Schulte, T. Allen, and A. El-Azab. Phase field modeling of the effect of porosity on grain growth kinetics in polycrystalline ceramics. Modelling and Simulation in Materials Science and Engineering, 21(6):065005, 2013.

[2] J. Ainscough, B. Oldfield, and J. Ware. Isothermal grain growth kinetics in sintered $\mathrm{UO}_{2}$ pellets. J. Nucl. Mater., 49(2):117-128, 1973.

[3] M. Ashby, J. Harper, and J. Lewis. The interaction of crystal boundaries with secondphase particles. Trans Met Soc AIME, 245(2):413-420, 1969.

[4] X. M. Bai and M. Li. Differences between solid superheating and liquid supercooling. Journal of Chemical Physics, 123(15), 2005. Bai, XM Li, M.

[5] C. B. Basak, A. K. Sengupta, and H. S. Kamath. Classical molecular dynamics simulation of uo2 to predict thermophysical properties. Journal of Alloys and Compounds, 360(1-2):210-216, 2003.

[6] A. L. Garcia, V. Tikare, and E. A. Holm. Three-dimensional simulation of grain growth in a thermal gradient with non-uniform grain boundary mobility. Scripta Materialia, 59(6):661 - 664, 2008.

[7] T. Gladman. On the theory of the effect of precipitate particles on grain growth in metals. Proceedings of the Royal Society of London. Series A. Mathematical and Physical Sciences, 294(1438):298-309, 1966.

[8] G. Gottstein and L. S. Shvindlerman. Grain boundary migration in metals: thermodynamics, kinetics, applications. CRC Press, Boca Raton, 1999.

[9] K. Govers, S. Lemehov, M. Hou, and M. Verwerft. Comparison of interatomic potentials for uo2: Part ii: Molecular dynamics simulations. Journal of Nuclear Materials, 376(1):66-77, 2008.

[10] P. Hellman and M. Hillert. On the effect of second-phase particles on grain growth. Scan. J. Met., 4:211, 1975. 
[11] M. Hillert. Inhibition of grain growth by second-phase particles. Acta Metallurgica, 36(12):3177-3181, 1988.

[12] Y. Liu and B. Patterson. Stereological analysis of zener pinning. Acta materialia, 44 (11):4327-4335, 1996.

[13] D. Manara, C. Ronchi, M. Sheindlin, M. Lewis, and M. Brykin. Melting of stoichiometric and hyperstoichiometric uranium dioxide. Journal of Nuclear Materials, 342 (13):148-163, 2005.

[14] M. I. Mendelev and D. J. Srolovitz. Impurity effects on grain boundary migration. Modelling Simul. Mater. Sci. Eng., 10:R79-109, 2002.

[15] M. I. Mendelev, C. Deng, C. A. Schuh, and D. J. Srolovitz. Comparison of molecular dynamics simulation methods for the study of grain boundary migration. Modelling and Simulation in Materials Science and Engineering, 21(4), 2013. Mendelev, M. I. Deng, C. Schuh, C. A. Srolovitz, D. J.

[16] P. C. Millett and M. Tonks. Meso-scale modeling of the influence of intergranular gas bubbles on effective thermal conductivity. J. Nucl. Mater., 412(3):281 - 286, 2011.

[17] N. Moelans, B. Blanpain, and P. Wollants. A phase field model for the simulation of grain growth in materials containing finely dispersed incoherent second-phase particles. Acta Materialia, 53(6):1771-1781, 2005.

[18] N. Moelans, B. Blanpain, and P. Wollants. Quantitative analysis of grain boundary properties in a generalized phase field model for grain growth in anisotropic systems. Phys. Rev. B, 78:024113, 2008.

[19] D. A. Molodov, U. Czubayko, G. Gottstein, and L. S. Shvindlerman. On the effect of purity and orientation on grain boundary motion. Acta Mater., 46(2):553-564, 1998.

[20] P. V. Nerikar, K. Rudman, T. G. Desai, D. Byler, C. Unal, K. J. McClellan, S. R. Phillpot, S. B. Sinnott, P. Peralta, B. P. Uberuaga, and C. R. Stanek. Grain boundaries in uranium dioxide: Scanning electron microscopy experiments and atomistic simulations. Journal of the American Ceramic Society, 94(6):1893-1900, 2011.

[21] G. Pastore, L. Swiler, J. Hales, S. Novascone, D. Perez, B. Spencer, L. Luzzi, P. Van Uffelen, and R. Williamson. Uncertainty and sensitivity analysis of fission gas behavior in engineering-scale fuel modeling. J. Nucl. Mater., Accepted, 2014.

[22] B. R. Patterson and Y. Liu. Quantification of grain boundary-pore contact during sintering. Journal of the American Ceramic Society, 73(12):3703-3705, 1990.

[23] S. J. Plimpton. Fast parallel algorithms for short-range molecular dynamics. J. Comp. Phys., 117:1-19, 1995. 
[24] D. Porter and K. Easterling. Phase Transformations in Metals and Alloys. Chapman and Hall, London, 2nd edition, 1992.

[25] W. T. Read and W. Shockley. Dislocation models of crystal grain boundaries. Physical Review, 78(3):275 - 289, 1950.

[26] C. Smith. Trans Met Soc AIME, 175:15-51, 1948.

[27] D. Srolovitz, M. Anderson, P. Sahni, and G. Grest. Computer-Simulation Of GrainGrowth .2. Grain-Size Distribution, Topology, And Local Dynamics. Acta Metallurgica, 32(5):793-802, 1984.

[28] M. Tonks, D. Gaston, P. Millett, D. Andrs, and P. Talbot. An object-oriented finite element framework for multiphysics phase field simulations. Comp. Mat. Sci., 51(1): 20-29, 2012.

[29] M. R. Tonks, P. C. Millett, P. Nerikar, S. Du, D. Andersson, C. R. Stanek, D. Gaston, D. Andrs, and R. Williamson. Multiscale development of a fission gas thermal conductivity model: Coupling atomic, meso and continuum level simulations. Journal of Nuclear Materials, 440(1):193-200, 2013.

[30] M. R. Tonks, Y. Zhang, S. Biner, P. C. Millett, and X. Bai. Guidance to design grain boundary mobility experiments with molecular dynamics and phase-field modeling. Acta Materialia, 61:1373-1382, 2013.

[31] M. R. Tonks, Y. Zhang, X. Bai, and P. C. Millett. Demonstrating the temperature gradient impact on grain growth in uo2 using the phase field method. Materials Research Letters, 2(1):23-28, 2014. doi: 10.1080/21663831.2013.849300.

[32] Z. T. Trautt, M. Upmanyu, and A. Karma. Interface mobility from interface random walk. Science, 314(5799):632-635, 2006.

[33] Z. T. Trautt, M. Upmanyu, and A. Karma. Interface mobility from interface random walk. Science, 314:632, 2006.

[34] M. Veshchunov. A new model of grain growth kinetics in $\{\mathrm{UO} 2\}$ fuel pellets. part 1: Grain growth kinetics controlled by grain face bubble migration. J. Nucl. Mater., 346 $(2-3): 208-219,2005$.

[35] D. Wolf. Structure-energy correlation for grain boundaries in fcc metals - i: boundaries on the (111) and (100) planes. Acta Metall., 37(7):1983 - 1993, 1989.

[36] D. Wolf. A read-shockley model for high-angle grain boundaries. Scripta Metall., 23: $1713-1718,1989$.

[37] H. Zhang, M. Upmanyu, and D. J. Srolovitz. Curvature driven grain boundary mi- 
gration in aluminum: molecular dynamics simulations. Acta Materialia, 53(1):79-86, 2005 .

[38] H. Zhang, M. Upmanyu, and D. J. Srolovitz. Curvature driven grain boundary migration in aluminum: molecular dynamics simulations. Acta Materialia, 53:79-86, 2005 .

[39] L. Zhang, M. R. Tonks, D. Gaston, J. W. Peterson, D. Andrs, P. C. Millett, and B. S. Biner. A quantitative comparison between $\mathrm{c} 0$ and $\mathrm{c} 1$ elements for solving the cahn-hilliard equation. Journal of Computational Physics, 2012.

[40] Y. Zhang, P. C. Millett, M. R. Tonks, X. M. Bai, and S. B. Biner. Molecular dynamics simulations of intergranular fracture in $\{\mathrm{UO} 2\}$ with nine empirical interatomic potentials. Journal of Nuclear Materials, 452(13):296 - 303, 2014. ISSN 0022-3115. 